\title{
A GLIMM-EFFROS DICHOTOMY FOR BOREL EQUIVALENCE RELATIONS
}

\author{
L. A. HARRINGTON, A. S. KECHRIS, AND A. LOUVEAU
}

\section{INTRODUCTION}

(A) A basic dichotomy concerning the structure of the orbit space of a transformation group has been discovered by Glimm [G12] in the locally compact group action case and extended by Effros [E1, E2] in the Polish group action case when additionally the induced equivalence relation is $F_{\sigma}$. It is the purpose of this paper to extend the Glimm-Effros dichotomy to the very general context of an arbitrary Borel equivalence relation (not even necessarily induced by a group action). Despite the totally classical descriptive set-theoretic nature of our result, our proof requires the employment of methods of effective descriptive set theory and thus ultimately makes crucial use of computability (or recursion) theory on the integers.

We consider Borel equivalence relations $E$ on Polish spaces $X$ (i.e., $E \subseteq X^{2}$ is Borel). A (countable) separating family for $E$ is a sequence $\left\{A_{n}\right\}$ of subsets of $X$ such that

$$
x E y \Leftrightarrow \forall n\left(x \in A_{n} \Leftrightarrow y \in A_{n}\right) .
$$

If the $A_{n}$ 's belong to some class $\Gamma$ we talk about a " $\Gamma$ separating family." Of particular interest of course is the case when the $A_{n}$ 's are Borel. If $E$ has a Borel separating family we say that $E$ is smooth. Notice that this is equivalent to saying that there is a Borel map $f: X \rightarrow Y, Y$ some Polish space, such that

$$
x E y \Leftrightarrow f(x)=f(y)
$$

so this means that there exist Borel calculable "invariants" $f(x)$, belonging to some Polish space, thus of a fairly "concrete" nature, associated with each $x \in X$ which classify $x$ up to $E$-equivalence. A typical example of such classification is the case $X=$ the $n \times n$ complex matrices $\left(=\mathbb{C}^{n^{2}}\right), E=$ the equivalence relation of similarity between $n \times n$ matrices, and $f(A)=$ the Jordan canonical form of $A$.

Received by the editors March 2, 1990.

1980 Mathematics Subject Classification (1985 Revision). Primary 03E15, 04A1 5, 46L05, 28 D99.

The first and second authors were partially supported by NSF grants.

(C) 1990 American Mathematical Society 
A selector for $E$ is a function $s: X \rightarrow X$ such that $s(x) E x$ and $x E y \Rightarrow$ $s(x)=s(y)$, while a transversal for $E$ is a set $T \subseteq X$ such that $T \cap[x]_{E}$ is a singleton for each equivalence class $[x]_{E}$ of $E$. Again we talk about "F selector" (resp. " $\Gamma$ transversal") if $s$ (resp. $T$ ) belongs to some class $\mathscr{F}$ (resp. $\Gamma)$. It is easy to check that the existence of a Borel selector for $E$ is equivalent to the existence of a Borel transversal and implies the smoothness of $E$. However, the converse fails even for closed equivalence relations.

A (finite, positive Borel) measure $\mu$ on $X$ is called (E-)ergodic if for every $\mu$-measurable $A \subseteq X$,

$$
A(E \text {-)invariant } \Rightarrow \mu(A)=0 \text { or } \mu(X-A)=0 .
$$

We call $\mu(E-)$ nonatomic if $\mu\left([x]_{E}\right)=0$ for each equivalence class $[x]_{E}$. Notice that if $E$ has an ergodic, nonatomic measure $\mu$, then $E$ is not smooth. (If $\left\{A_{n}\right\}$ is a Borel, more generally, $\mu$-measurable separating family, each $A_{n}$ is invariant. Then $A=\bigcap_{n}\left\{A_{n}: \mu\left(X-A_{n}\right)=0\right\} \cap \bigcap_{n}\left\{X-A_{n}: \mu\left(A_{n}\right)=0\right\}=[x]_{E}$ for some $x$, but on the other hand $A$ has full measure.)

A typical example of a nonsmooth equivalence relation is the following: Let $\omega=\{0,1,2, \ldots\}$ and $X=2^{\omega}$ be the space of infinite binary sequences with the product topology. Let $E_{0}$ be defined by

$$
x E_{0} y \Leftrightarrow \exists n \forall m \geq n(x(m)=y(m)) .
$$

As the standard product measure on $2^{\omega}$ (with $2=\{0,1\}$ given the $(1 / 2,1 / 2$ ) measure) is $E_{0}$-ergodic and nonatomic, $E_{0}$ is not smooth.

One final notion before we state our main result. Given equivalence relations $(X, E)$ and $\left(X^{\prime}, E^{\prime}\right)$, a reduction of $E$ into $E^{\prime}$ is a map $f: X \rightarrow X^{\prime}$ such that $x E y \Leftrightarrow f(x) E^{\prime} f(y)$. In particular the induced map

$$
\tilde{f}\left([x]_{E}\right)=[f(x)]_{E^{\prime}}
$$

maps $X / E 1-1$ into $X^{\prime} / E^{\prime}$. It $f$ itself is $1-1$ we say that $f$ is an embedding of $E$ into $E^{\prime}$. Put

$$
\begin{aligned}
& E \leq E^{\prime} \Leftrightarrow \text { there is a Borel reduction of } E \text { into } E^{\prime}, \\
& E \sqsubseteq E^{\prime} \Leftrightarrow \text { there is a Borel embedding of } E \text { into } E^{\prime} .
\end{aligned}
$$

For example, if $\Delta(X)$ is the equality relation on $X$ and $\Delta:=\Delta\left(2^{\omega}\right)$ then $E$ is smooth iff $E \leq \Delta$.

Intuitively speaking $E \leq E^{\prime}$ means that we can classify elements of $X$ up to $E$-equivalence by invariants which are $E^{\prime}$-equivalence classes, i.e., members of $X^{\prime} / E^{\prime}$ (which in general will not be concrete). A typical such reduction occurs in the spectral theorem. In a particular instance it classifies multiplicity-free normal operators on Hilbert space with respect to unitary equivalence $(E)$ by measures on $\mathbb{C}$ with respect to mutual absolute continuity $\left(E^{\prime}\right)$, so that the invariants are now measure classes on $\mathbb{C}$.

We can state now our main result. 
Theorem 1.1. Let $X$ be a Polish space and $E$ a Borel equivalence relation on $X$. Then exactly one of the following holds:

(I) $E$ is smooth or $E$.

(II) $E_{0} \sqsubseteq E$ (continuously), i.e., there is a continuous embedding of $E_{0}$ into

Moreover in (I) we can replace "smooth," i.e., the existence of a Borel separating family, by the existence of a universally measurable separating family and in (II) the existence of a continuous embedding of $E_{0}$ into $E$ by the existence of a universally measurable embedding.

Also, $(\mathrm{I})$ is equivalent to

$(\mathrm{I})^{*} E$ has a selector which is $C$-measurable, i.e., measurable with respect to the smallest $\sigma$-algebra containing the open sets and closed under the Souslin operation $\mathscr{A}$,

as well as to

(I)** There is a Polish topology $\sigma$ extending that of $X$ such that $E$ is closed in $\left(X^{2}, \sigma \times \sigma\right)$.

Finally, (II) is equivalent to

(II)* There is a nonatomic, ergodic measure for $E$.

Here are a few corollaries of this result. It is well known and easy to see that every $G_{\delta}$ equivalence relation has an analytic separating family. So we have

Corollary 1.2. Let $E$ be a $G_{\delta}$ equivalence relation on a Polish space $X$. Then $E$ is smooth, so in particular there is a Polish topology $\sigma$ extending that of $X$ such that $E$ is closed in $\left(X^{2}, \sigma \times \sigma\right)$.

Thus $G_{\delta}$ and closed equivalence relations have the same structure.

As we already mentioned, a smooth closed equivalence relation might not have a Borel selector. However, by standard uniformization theorems, we have that if $E$ is a Borel equivalence relation on a Polish space $X$ and each equivalence class is $K_{\sigma}$ (i.e., a countable union of compact sets), then $E$ is smooth iff $E$ has a Borel selector. For Borel equivalence relations with $K_{\sigma}$ classes we can then add to (I) the following equivalent

$(\mathrm{I})^{* * *} E$ has a Borel selector.

Examples of such $E$ are the countable ones (i.e., those for which each equivalence class is countable) and those induced by actions of second countable locally compact transformation groups.

Given now $E$, a Borel equivalence relation on a Polish space $X$, and a Borel set $A \subseteq X$ we call $A(E$-)smooth if $E\lceil A$ has a Borel separating family (i.e., there is a sequence of Borel sets $\left\{A_{n}\right\}$ such that for $x, y \in A, x E y \Leftrightarrow$ $\left.\forall n\left[x \in A_{n} \Leftrightarrow y \in A_{n}\right]\right)$. (Thus $E$ is smooth iff $X$ is smooth.) Then we have the following characterization of Borel smooth sets extending results of ShelahWeiss [SW] and Weiss [W] who dealt with the case of hyperfinite $E$ (that is $E$ induced by the orbits of a single Borel automorphism of $X$, i.e., by a Borel $\mathbb{Z}$-action). 
Corollary 1.3. Let $E$ be a Borel equivalence relation on a Polish space $X$. Then the following are equivalent for a Borel set $A \subseteq X$ :

(i) $A$ is smooth.

(ii) For every nonatomic, ergodic measure $\mu$ on $X, \mu(A)=0$.

One can extend these concepts and results also to analytic sets. The notion of smoothness as a notion of smallness and its definability properties are studied in C. Uzcategui's Ph.D. thesis [U].

Finally let us mention that although the combinatorial structure of $E_{0}$ plays an essential role in the proof of Theorem 1.1, alternative (II) (with just Borel instead of continuous embeddings) will still hold for a much wider class of equivalence relations instead of $E_{0}$. More precisely, $E_{0}$ can be replaced there by any nonsmooth hyperfinite Borel equivalence relation $E^{*}$. This is because a theorem of Dougherty-Jackson-Kechris [DJK2] asserts that any two nonsmooth, hyperfinite Borel equivalence relations are embeddable into each other.

(B) Before we discuss the effective descriptive set theoretic approach to the proof of Theorem 1.1, we will review some of the earlier work that led to our results here. The dichotomy expressed in Theorem 1.1 was first discovered by Glimm [Gl2] (in a paper that grew out of his earlier work [Gl1] on the proof of the Mackey "smooth dual iff type I" conjecture in the representation theory of $C^{*}$-algebras and groups) for the case where $E$ is the equivalence relation induced by the orbits of a second countable locally compact transformation group. Effros [E1, E2] then extended the Glimm results to the case of $E$ induced by a Polish transformation group, provided that moreover $E$ is $F_{\sigma}$. In [E1] (see also [E2]) this extension is applied to give a new and simpler proof of the Mackey conjecture. In these Glimm, Effros papers it is shown for instance that for the kind of equivalence relations the authors are dealing with, exactly one of the following holds:

(I) $E$ is smooth, equivalently

(II)*** $E$ has a Borel selector, or

$(\mathrm{I})^{* * * *}$ Every equivalence class is locally closed (i.e., the difference of two closed sets), or else

(II)* There is a nonatomic, ergodic measure for $E$.

(In fact these papers contain a number of other conditions equivalent to (I).) Although our main formulation of the second alternative in the form

(II) $E_{0} \sqsubseteq E$ (continuously)

is not explicitly isolated in these papers, it is surely present in the proofs of these results and the role of $E_{0}$ as the prototype of the nonsmooth behaviour is pointed out in the opening paragraph of [Gl2] (see also Dixmier [D, 9.5.5] and Zimmer [Z, p. 13]).

In Godefroy [Go] it is shown that $(\mathrm{I}),(\mathrm{I})^{* * * *}$ are equivalent for any $E \in F_{\sigma}$ 
which is induced by a group of homeomorphisms, while in Burgess [B2] it is shown that $(\mathrm{I}),(\mathrm{I})^{* * *}$ are equivalent for any $E$ induced by a Polish transformation group.

In the papers by Katznelson-Weiss [KW], Krieger [Kr1, Kr2], Shelah-Weiss [SW], and Weiss [W] various instances of Glimm-Effros dichotomy are established and applied in ergodic theory. In the most recent by Weiss [W] the author essentially establishes the dichotomy (I) or (II) for hyperfinite Borel equivalence relations (in a somewhat stronger form in this case). Finally, in the unpublished [DJK1] the authors prove the dichotomy (I) (but with an analytic instead of Borel separating family) or (II) for arbitrary $F_{\sigma}$ equivalence relations, thereby eliminating any group action hypotheses.

(C) The second line of work leading to our results originates in the theorem of Silver [S] concerning coanalytic equivalence relations and a subsequent proof of that result by Harrington. Silver's theorem (which we believe was originally conjectured by $\mathrm{H}$. Friedman) asserts the following: Let $E$ be a coanalytic equivalence relation on a Polish space $X$. Then $X / E$ is either countable or else has cardinality $2^{\aleph_{0}}$. In fact if it is uncountable, then there is a perfect set $P \subseteq X$ of pairwise $E$-inequivalent elements. This is in particular true for Borel $E$, a fact which is also easily a consequence of Theorem 1.1. For the case when $E$ is analytic one can only say that $X / E$ has cardinality at most $\aleph_{1}$ or else $2^{\aleph_{0}}$, by a theorem of Burgess [B1]. However, it is an open problem to decide if the assertion " $|X / E| \leq \aleph_{0}$ or $|X / E|=2{ }^{\aleph_{0}}$ " is true when $E$ is induced by the orbits of a Polish transformation group. That this holds is known as the topological Vaught conjecture and it implies the famous Vaught conjecture in model theory, which asserts that every first-order theory (in a countable language) has either countably many or else $2^{\mathrm{N}_{0}}$ countable models (up to isomorphism).

Harrington (unpublished) later gave a much simpler proof of Silver's Theorem, which made crucial use of the methods of effective descriptive set theory. (Readers not familiar with this subject will find an elementary introduction in [MK] and $\S 3$ of this paper.) In his proof Harrington employed the topology generated by the effectively analytic $\left(\Sigma_{1}^{1}\right)$ sets, a concept first introduced much earlier by Gandy but used rather sparingly before Harrington's application. Actually both Gandy and Harrington utilize the equivalent language of forcing, but for purposes of communicating these arguments to nonlogicians the topological approach is much more convenient. One can find a write-up (which owes much to an earlier set of seminar notes by Louveau) of Harrington's proof in topological language in the expository paper [MK].

After this first application, the Gandy-Harrington topology has become a standard tool in the study of Borel structures. For instance it has been used to develop an extensive structure theory for Borel orderings in Harrington-MarkerShelah [HMS], Louveau [L], Louveau-Saint Raymond [LS], and Kada [Ka]. It is also the main tool through which effective descriptive set theory is applied in our 
paper. In particular, we use some of the techniques developed in HarringtonMarker-Shelah [HMS].

(D) In the spirit of effective descriptive set theory, Theorem 1.1 will be proved by establishing a stronger effective version. (Below $\Delta_{1}^{1}$ is the effective analog of analytic-coanalytic, i.e., Borel-this will be defined precisely in $\S 3$.) We denote by $\mathscr{N}$ the Polish space $\mathscr{N}=\omega^{\omega}$ of all infinite sequences $x: \omega \rightarrow \omega$ with the product topology, $\omega$ being discrete.

Theorem 1.4. Let $E$ be a $\Delta_{1}^{1}$ equivalence relation on $\mathcal{N}$. Then exactly one of the following holds:

(I) $E$ has a separating family $\left\{A_{n}\right\}$ consisting of $\Delta_{1}^{1}$ sets (in fact uniformly, i.e., there is a separating family $\left\{A_{n}\right\}$ such that the set $(x, n) \in A \Leftrightarrow x \in A_{n}$ is $\Delta_{1}^{1}$ in $\left.\mathscr{N} \times \omega\right)$ or

(II) $E_{0} \sqsubseteq E$ (continuously).

We restrict attention here to the standard model $\mathscr{N}$ as it is more convenient for the development of the effective theory that we will outline in $\S 3$. As we will see in $\S 5$ one can easily obtain from that not only Theorem 1.1 for $X=\mathscr{N}$ but for arbitrary Polish $X$ as well by using standard "transfer" arguments-after all any two uncountable Polish spaces are Borel isomorphic!

(E) One can also view Silver's Theorem (for Borel $E$ ) and Theorem 1.1 as providing the first steps toward understanding the structure of the partial preorders $E \leq F, E \sqsubseteq F$ of reducibility or embedding. For Borel equivalence relations $E$ with $X / E$ uncountable, Silver's theorem asserts that $\Delta$ is a minimum in $\leq$ (and $\sqsubseteq$ ) and our result states that $E_{0}$ is least (in $\leq$ or $\sqsubseteq$ ) above $\Delta$. Beyond that the picture is quite unclear at this stage: Already the structure of countable Borel $E$ (i.e., Borel $E$ with each equivalence class countable) under $\leq$ or $\sqsubseteq$ is quite intriguing. A first study of such $E$ 's, which are of particular interest as they naturally arise in ergodic theory, operator algebras, as well as certain areas of logic like recursion theory, has been undertaken in [Ke] and (the unpublished) [DJK2].

(F) We will finally discuss the organization of this paper. We have made an attempt to make it as self-contained as possible, especially for readers not familiar with the concepts and results of effective descriptive set theory. We assume however familiarity with the classical descriptive set theory of Borel and analytic sets, as in Kuratowski [Ku] for example.

In $\S 2$ we present some standard material concerning the topological Choquet games. These provide a most convenient vehicle for the application of Baire category type arguments that will be essential in our proof. For the classical general theory of Baire category on topological spaces we refer again to Kuratowski [Ku].

In $\S 3$ we review effective descriptive set theory. Again the material is all standard and can be found with the appropriate references and historical remarks 
in Moschovakis [M]. However we do not assume that the reader is familiar with $[\mathrm{M}]$. We have originally toyed with the idea of presenting a totally self-contained development of effective descriptive set theory leading to a proof of the results that we will need, but soon realized this would make the paper unrealistically long. So we settled on a compromise. Since there is a fairly elementary and rather short introduction to the subject given in $[\mathrm{MK}]$, we refer the reader to that paper, especially $\S \S 6$ and 7 , for a first acquaintance with the subject. In $\S 3$ we present then a quick review of that material giving all definitions but skipping proofs that can be found in $[\mathrm{MK}]$. We then provide some theorems not given in $[\mathrm{MK}]$ that we will need, with complete proofs.

In $\S 4$ we introduce the Gandy-Harrington topology and prove the basic facts about it that we will use later. (The reader can probably profit by reading $\S 9$ of [MK], where Harrington's proof of Silver's theorem is given.)

Finally, in $\S 5$ we give the proof of the effective Theorem 1.4 and deduce from this Theorem 1.1 and its corollaries.

\section{Choquet Games AND SPACES}

We will review here some standard facts concerning topological games introduced by Choquet in relation to Baire category arguments.

Let $X$ be a topological space. The Choquet game $C_{X}$ associated to $X$ is played as follows: Players $\alpha, \beta$ take turns, with $\beta$ playing first, choosing non$\varnothing$ open subsets of $X$, so that each open set played by any player is contained in the opponent's previous move.

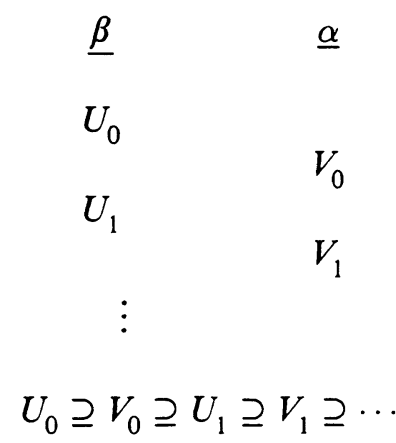

We say that $\alpha$ wins a run of this game $U_{0}, V_{0}, U_{1}, V_{1}, \ldots$ if $\bigcap_{n} V_{n}$ $\left(=\bigcap_{n} U_{n}\right) \neq \varnothing$. A winning strategy for $\alpha$ is a rule that tells him how to play, as he sees his opponent's moves, so that he wins every run of the game in which he follows this strategy. Formally, it is a sequence of functions $f_{1}, \ldots, f_{n}, \ldots$ with $f_{n}:\left(\mathscr{T}^{*}\right)^{n} \rightarrow \mathscr{T}^{*}$, where $\mathscr{T}^{*}$ is the class of non- $\varnothing$ open subsets of $X$, such that $f\left(U_{0}, \ldots, U_{n-1}\right) \subseteq U_{n-1}$ and for any $U_{0}, U_{1}, U_{2}, \ldots$ if $f\left(U_{0}, \ldots, U_{n-1}\right)=V_{n-1}$ and $U_{0} \supseteq V_{0} \supseteq U_{1} \supseteq V_{1} \cdots$ holds then $\bigcap_{n} V_{n} \neq \varnothing$.

We say that $X$ is a Choquet space if $\alpha$ has a winning strategy in the Choquet game of $X$.

A variation of the Choquet game will actually be more useful to us. Given $X$, the strong Choquet game $C_{X}^{*}$ is played as follows: Players $\alpha, \beta$ take turns, 
with $\beta$ playing first, playing non- $\varnothing$ open subsets as before, except that $\beta$ also plays a point in his open set at each move and $\alpha$ must then play an open set containing this point.

$\begin{array}{cr}\underline{\beta} & \underline{\alpha} \\ U_{0}, x_{0} & \\ U_{1}, x_{1} & V_{0} \\ & V_{1} \\ x_{i} \in U_{i}, & x_{i} \in V_{i} \\ U_{0} \supseteq V_{0} \supseteq U_{1} \supseteq V_{1} \supseteq \cdots\end{array}$

Again $\alpha$ wins iff $\bigcap_{n} V_{n} \neq \varnothing$. The notion of a winning strategy for $\alpha$ is defined in a similar way as before and we say that $X$ is a strong Choquet space if $\alpha$ has a winning strategy in the strong Choquet game for $X$.

We will need the following standard facts:

Proposition 2.1. (i) A strong Choquet space is a Choquet space. A Choquet space is a Baire space (i.e., the intersection of countably many open dense sets is dense).

(ii) If $X, Y$ are (strong) Choquet spaces, so is $X \times Y$.

(iii) If $X$ is a strong Choquet space and $G \subseteq X$ is a $G_{\delta}$ set in $X$, then $G$ (with the relative topology) is a strong Choquet space.

Proof. (i) The first statement is obvious. For the second, let $G_{n}$ be dense open, and $U$ open non- $\varnothing$. We want to show $\bigcap_{n} G_{n} \cap U \neq \varnothing$. Play the following run of the Choquet game: $\beta$ starts with $G_{0} \cap U=U_{0}$ and $\alpha$ answers by his winning strategy to play $V_{0}$. II plays then $G_{1} \cap V_{0}=U_{1}$ and $\alpha$ answers by $V_{1}$, etc. Clearly $\varnothing \neq \bigcap_{n} V_{n} \subseteq \bigcap_{n} G_{n} \cap U$.

(ii) This is straightforward, since every non- $\varnothing$ open set in $X \times Y$ is the union of rectangles $U \times V$ with $U, V$ open in $X, Y$ resp.

(iii) Let $G=\bigcap_{n} G_{n}$, with $G_{n}$ open. Denote by $\Sigma$ a winning strategy for $\alpha$ in the strong Choquet game for $X$, which we denote by $C$. Denote by $C^{\prime}$ the strong Choquet game on $G$ and by $\alpha^{\prime}, \beta^{\prime}$ its players. We will describe a winning strategy for $\alpha^{\prime}$ in $C^{\prime}$. Let $\beta^{\prime}$ start with $\left(U_{0}^{\prime}, x_{0}^{\prime}\right)$. Let $U_{0}$ be open in $X$ with $G \cap U_{0}=U_{0}^{\prime}$. Let $\beta$ play $\left(U_{0} \cap G_{0}, x_{0}^{\prime}\right)$ in $C$ and $\alpha$ answer, using $\Sigma$, by $V_{0} \subseteq G_{0}$. As $x_{0}^{\prime} \in V_{0}, V_{0}^{\prime}=V_{0} \cap G \neq \varnothing$; $\alpha^{\prime}$ answers $\left(U_{0}^{\prime}, x_{0}^{\prime}\right)$ by playing $V_{0}^{\prime}$. Next $\beta$ plays $\left(U_{1}^{\prime}, x_{1}^{\prime}\right)$. Again let $U_{1}$ be open in $X$ with $G \cap U_{1}=U_{1}^{\prime}$. Replacing $U_{1}$ by $U_{1} \cap V_{0}$ if necessary, we can assume $U_{1} \subseteq V_{0}$. So $\left(U_{1} \cap G_{1}, x_{1}^{\prime}\right)$ is a legitimate next move for $\beta$ in $C$; $\alpha$ answers it by $\Sigma$ playing $V_{1} \subseteq G_{1}$. As $x_{1}^{\prime} \in V_{1}, V_{1}^{\prime}=V_{1} \cap G \neq \varnothing$. So $\alpha^{\prime}$ answers $\left(U_{1}^{\prime}, x_{1}^{\prime}\right)$ by playing $V_{1}^{\prime}$. Proceed this way ad infinitum. Clearly $\bigcap_{n} V_{n} \neq \varnothing$. But $\bigcap_{n} V_{n} \subseteq \bigcap_{n} G_{n}=G$, so $\bigcap_{n}\left(V_{n} \cap G\right)=\bigcap_{n} V_{n}^{\prime} \neq \varnothing$ and $\alpha^{\prime}$ wins.

The following simple observation will be convenient later on: Let $d$ be a 
metric on $X$, and denote the $d$-diameter of a set $A \subseteq X$ by $\operatorname{diam}(A)$. If every $d$-open set is open in $X$ and $X$ is a (strong) Choquet space then we can assume that in the (strong) Choquet game of $X, \alpha$ has a winning strategy which guarantees that $\operatorname{diam}\left(V_{n}\right)<1 /(n+1)$, so that in particular $\bigcap_{n} V_{n}$ is a singleton.

To see this let $g_{n}$ be a function such that for each non- $\varnothing$ open set $U$ of $X, g_{n}(U)$ is a non- $\varnothing$ open subset of $U$ of diameter $<1 /(n+1)$. If $\left\{f_{n}\right\}$ is a winning strategy for $\alpha$ in the Choquet game, let $f_{n}^{\prime}\left(U_{0}, U_{1}, \ldots, U_{n}\right)=$ $f_{n}\left(U_{0}, U_{1}, \ldots, g_{n}\left(U_{n}\right)\right)$. This new strategy clearly works. For the strong Choquet game let

$f_{n}^{\prime}\left(\left(U_{0}, x_{0}\right), \ldots,\left(U_{n}, x_{n}\right)\right)=f_{n}\left(\left(U_{0}, x_{0}\right), \ldots,\left(U_{n} \cap S\left(x_{n}, 1 /(n+1)\right), x_{n}\right)\right)$,

where $S(x, \epsilon)=\{y: d(x, y)<\epsilon\}$.

\section{EFFECTIVE DESCRIPTIVE SET THEORY}

3.1. Basic notions. First we will quickly review some basic notions and facts of effective descriptive set theory which are all explained in detail in [MK].

Let $\omega=\{0,1,2, \ldots\}$ be the space of nonnegative integers with the discrete topology and $\mathscr{N}=\omega^{\omega}$ the Baire space, i.e., the space of infinite sequences $x: \omega \rightarrow \omega$ from $\omega$ with the product topology. For a finite sequence $(s(0), \ldots, s(n-1))=s \in \omega^{<\omega}$ let $N_{s}=\left\{x \in \omega^{\omega}: s \subseteq x\right\} \quad(s \subseteq x$ means $s(i)=x(i), \forall i<n)$. Then $\left\{N_{s}\right\}$ is the standard basis for the topology of $\mathscr{N}$. The spaces $\mathscr{X}=X_{1} \times \cdots \times X_{k}$, where $X_{i}=\omega$ or $X_{i}=\mathscr{N}$, will be equipped with the product topology. They are clearly homeomorphic to $\mathscr{N}$ if at least one $X_{i}$ is to $\mathscr{N}$ and otherwise to $\omega$. Taking the class of singletons $\{n\}, n \in \omega$, as the standard basis for $\omega$ we can define a standard basis for each product space in the obvious way. For $\mathscr{X}=\omega \times \mathscr{N}$ for instance this will be the collection of all $\{n\} \times N_{s}$.

We introduce now the notions of effective topology on these product spaces. (It is assumed that the reader is familiar with the concept of a recursive (i.e., computable) function $f: \omega^{n} \rightarrow \omega$ (see [MK] for a quick review). The notion can be straightforwardly extended to functions on spaces such as $\omega^{<\omega}$ via some canonical bijection of $\omega^{<\omega}$ with $\omega$.)

A set $A \subseteq \mathscr{X}$ is effectively open or $\Sigma_{1}^{0}$ iff it is an effective (computable) union of basic open sets in $\mathscr{Z}$.

Spelled out in detail for $\mathscr{X}=\omega \times \mathscr{N}$, for example, this means that $A=$ $\bigcup_{n}\left\{i_{n}\right\} \times N_{s_{n}}$, where $n \mapsto i_{n}, n \mapsto s_{n}$ are recursive functions. Since there are only countably many recursive functions there are only countably many $\Sigma_{1}^{0}$ sets.

A function $f: \mathscr{X} \rightarrow \mathscr{N}$ is effectively continuous or recursive if $f^{-1}\left[N_{s}\right]$ is $\Sigma_{1}^{0}$ uniformly on $s$, i.e., the set

$$
G_{f}(x, s) \Leftrightarrow x \in f^{-1}\left[N_{s}\right]
$$

is $\Sigma_{1}^{0}$ in $\mathscr{Z} \times \omega$. 
Similarly $f: \mathscr{X} \rightarrow \omega$ is recursive if $G_{f}(x, n) \Leftrightarrow f(x) \in\{n\} \Leftrightarrow f(x)=$ $n$ is $\Sigma_{1}^{0}$. (This agrees with the usual definition for $\mathscr{X}=\omega^{n}$.) In general, $f: \mathscr{X} \rightarrow \mathscr{Y}=Y_{1} \times \cdots \times Y_{k}$ is recur sive if $p_{i} \circ f, 1 \leq i \leq k$, are recursive, where $p_{i}: Y \rightarrow Y_{i}$ is the $i$ th projection. Again there are only countably many recursive functions.

One can easily verify that $\Sigma_{1}^{0}$ is closed under finite intersections and unions, recursive preimages, and projections. (It is clearly not closed under countable unions.) Note also that every $\mathscr{X}=X_{1} \times \cdots \times X_{n}$ with at least one $X_{i}=\mathscr{N}$ is recursively homeomorphic to $\mathscr{N}$.

A set $A \subseteq \mathscr{Z}$ is effectively closed or $\Pi_{1}^{0}$ if $\mathscr{Z}-A$ is $\Sigma_{1}^{0}$. Then let $A \subseteq \mathscr{Z}$ be effectively analytic or $\Sigma_{1}^{1}$ if $A$ is the projection of a $\Pi_{1}^{0}$ set on $\mathscr{X} \times \mathscr{N}$, i.e., for some $B \in \Pi_{1}^{0}, B \subseteq X \times \mathscr{N}$,

$$
x \in A \Leftrightarrow \exists y(x, y) \in B .
$$

Finally, $A \subseteq \mathscr{X}$ is effectively coanalytic or $\Pi_{1}^{1}$ iff $\mathscr{Z}-A$ is $\Sigma_{1}^{1}$. A set which is both $\Sigma_{1}^{1}$ and $\Pi_{1}^{1}$ will be called a $\Delta_{1}^{1}$ set.

Again it is not hard to verify that $\Sigma_{1}^{1}$ is closed under finite intersections and unions, preimages by $\Delta_{1}^{1}$ functions (i.e., functions with $\Delta_{1}^{1}$ graphs), and projections. It is also closed under coprojections over $\omega$, i.e., if $A \subseteq \mathscr{X} \times \omega$ is $\Sigma_{1}^{1}$ so is $B \subseteq \mathscr{X}$, where

$$
x \in B \Leftrightarrow \forall n(x, n) \in A .
$$

Also $\Pi_{1}^{1}$ is closed under finite intersections and unions, $\Delta_{1}^{1}$ preimages, and coprojections, as well as projections over $\omega$. Finally $\Delta_{1}^{1}$ is closed under finite intersections and unions, $\Delta_{1}^{1}$ preimages, projections, and coprojections over $\omega$.

There is a simple but very useful representation of $\Pi_{1}^{0}$ and $\Sigma_{1}^{0}$ sets that we proceed to describe. By a tree on $\omega$ we mean a set $T \subseteq \omega^{<\omega}$ of finite sequences closed under initial segments, i.e., $s \subseteq t \in T \Rightarrow s \in T(s \subseteq t$ means $s=(s(0), \ldots, s(n-1)), t=(t(0), \ldots, t(m-1))$, where $n \leq m$ and $s(i)=t(i) \quad \forall i<n)$. The body of the tree $T$, in symbols [T], is the set of all infinite branches through $T$, i.e.,

$$
[T]=\{x \in \mathscr{N}: \forall n(x \mid n \in T)\} .
$$

Clearly the sets of the form $[T]$ are the closed subsets of $\mathscr{N}$. Similarly we define the notion of a tree on $\omega \times \omega$ as being a set of pairs of same length finite sequences $(s, t) \in \omega^{<\omega} \times \omega^{<\omega}$ such that if $s \subseteq u, t \subseteq v,(u, v) \in T$, and $s, t$ have the same length, then $(s, t) \in T$. Again let $[T]=\left\{(x, y) \in \mathscr{N}^{2}: \forall n\right.$ $\left(x\lceil n, y\lceil n) \in T\}\right.$. The sets $[T]$ are again the closed subsets of $\mathscr{N}^{2}$. Similar remarks apply to $\mathcal{N}^{n}$ for all $n$.

We have now that $A \subseteq \mathscr{N}$ is $\Pi_{1}^{0}$ iff $A=[T]$, where $T$ is a recursive tree and $A \subseteq \mathscr{N}$ is $\Sigma_{1}^{1}$ iff $A=p[T]$, where $T$ is a recursive tree on $\omega \times \omega$ and $p: \mathscr{N}^{2} \rightarrow \mathscr{N}$ denotes the projection to the first coordinate, i.e., $A=\{x$ : $\exists y(x, y) \in[T]\}$. 
3.2. Universal sets and uniformities. A fundamental property of the classes $\Sigma_{1}^{0}, \Pi_{1}^{0}, \Sigma_{1}^{1}$, and $\Pi_{1}^{1}$ is the existence of universal sets. Given $U \subseteq \mathscr{Z} \times \mathscr{Y}$ and $x \in \mathscr{X}$ denote by $U_{x}=\{y:(x, y) \in U\}$ the $x$ section of $U$. We now have the following:

Let $\Gamma$ be any of $\Sigma_{1}^{0}, \Pi_{1}^{0}, \Sigma_{1}^{1}, \Pi_{1}^{1}$. Given a product space $\mathscr{Z}$ there is $U^{\mathscr{L}} \subseteq$ $\omega \times \mathscr{X}$ which is universal for $\Gamma$, i.e., $U^{\mathscr{Z}} \in \Gamma$ and for any $A \subseteq \mathscr{X}: A \in \Gamma$ iff $\exists n \in \omega\left(A=U_{n}^{\mathscr{Q}}\right)$. For a fixed $\Gamma$ as above we call $\left\{U^{\mathscr{L}}\right\}$ a universal system for $\Gamma$. Clearly if $\left\{U^{\mathscr{L}}\right\}$ is a universal system for $\Sigma_{1}^{1}$, for example, $\left\{\omega \times \mathscr{Z}-U^{\mathscr{L}}\right\}$ is one for $\Pi_{1}^{1}$.

Given $\left\{U^{\mathscr{X}}\right\}$ a universal system for $\Gamma$ and $A \subseteq \mathscr{X}$ in $\Gamma$ we view any $n \in \omega$ such that $A=U_{n}^{\mathscr{D}}$ as a code of $A$. (A set has in general infinitely many codes.) We have already seen various basic closure properties of the classes $\Gamma$ above. For example if $A, B \subseteq \mathscr{X}$ are $\Sigma_{1}^{1}$ so is $A \cap B$. An additional consideration now arises. Given codes $n, m$ for $A, B$ resp. can we determine effectively a code for $A \cap B$ ? This is extremely desirable in a lot of situations. Note that our coding depends on choosing a universal system $\left\{U^{\mathscr{L}}\right\}$, which is by no means unique. There are "bad" universal systems for which the above desired effectiveness or uniformity, as it is usually called, may fail. But we can always find "good" universal systems and this is the context of the next theorem. Its statement looks a little ad hoc at first but we will see shortly how it achieves our goals of uniformity.

Theorem 3.2.1 (Existence of good universal systems). Let $\Gamma$ be any of $\Sigma_{1}^{0}, \Pi_{1}^{0}$, $\Sigma_{1}^{1}, \Pi_{1}^{1}$. There is a universal system for $\Gamma,\left\{U^{\mathscr{L}}\right\}$, with the following property. For any $m$ and product space $\mathscr{X}$ there is a recursive function $S^{m, \mathscr{Z}}: \omega^{m+1} \rightarrow \omega$ such that

$$
\left(e, k_{1}, \ldots, k_{m}, x\right) \in U^{\omega^{m} \times \mathscr{Z}} \Leftrightarrow\left(S^{m, \mathscr{X}}\left(e, k_{1} \cdots k_{m}\right), x\right) \in U^{\mathscr{L}} .
$$

Proof. Let $\left\{V^{\mathscr{L}}\right\}$ be any universal system for $\Gamma$. Fix, for each $k$, a recursive bijection $f_{k}$ between $\omega^{k}$ and $\omega$ and put for convenience $f_{k}\left(n_{0}, \ldots, n_{k-1}\right)=$ $\left\langle n_{0}, \ldots, n_{k-1}\right\rangle$ and $n=\left\langle(n)_{0}, \ldots,(n)_{k-1}\right\rangle$. Define $U^{\mathscr{L}}$ by

$$
(e, x) \in U^{\mathscr{L}} \Leftrightarrow\left((e)_{0},(e)_{1}, x\right) \in V^{\omega \times \mathscr{L}} .
$$

Easily $\left\{U^{\mathscr{X}}\right\}$ is a universal system. Now for fixed $m, \mathscr{X}$ let $Q_{\mathscr{X}}^{m} \subseteq \omega \times \mathscr{X}$ be defined by

$$
(e, x) \in Q_{\mathscr{L}}^{m} \Leftrightarrow\left((e)_{0}, \ldots,(e)_{m}, x\right) \in U^{\omega^{m} \times \mathscr{L}} .
$$

Then $Q_{\mathscr{X}}^{m}$ is in $\Gamma$, so for some $e_{0}$ (depending on $m, \mathscr{X}$ )

$$
(e, x) \in Q_{\mathscr{Z}}^{m} \Leftrightarrow\left(e_{0}, e, x\right) \in V^{\omega \times \mathscr{X}} .
$$

Put finally

$$
S^{m, \mathscr{L}}\left(e, k_{1} \cdots k_{m}\right)=\left\langle e_{0},\left\langle e, k_{1} \cdots k_{m}\right\rangle\right\rangle \text {. }
$$


From now on we fix for each class $\Gamma$ as above a good universal system $\left\{U_{\Gamma}^{\mathscr{L}}\right\}$ for $\Gamma$. We can assume that $U_{\Pi_{1}^{1}}^{\mathscr{L}}=\omega \times \mathscr{X}-U_{\Sigma_{1}^{1}}^{\mathscr{X}}$. We also fix the functions $S_{\Gamma}^{m, \mathscr{X}}$ as above. Again $S_{\Sigma_{1}^{1}}^{m, \mathscr{Z}}=S_{\Pi_{1}^{1}}^{m, \mathscr{L}}$. We will usually omit some or all of these superscripts and subscripts when there is no danger of confusion. Codes for sets in $\Gamma$ will always be relative to these fixed systems. Let us see now how the various closure properties of $\Gamma$ hold "effectively in the codes": Take for example intersection. Fix $\mathscr{X}$. We want to find a recursive function $f: \omega^{2} \rightarrow \omega$ such that if $n$ is any code of $A \subseteq \mathscr{X}$ and $m$ is any code of $B \subseteq \mathscr{X}$ then $f(m, n)$ is a code of $A \cap B$, i.e., $U_{m}^{\mathscr{Z}} \cap U_{n}^{\mathscr{Z}}=U_{f(m, n)}^{\mathscr{L}}$. To find $f$ put

$$
(m, n, x) \in C \Leftrightarrow(m, x) \in U^{\mathscr{X}} \text { and }(n, x) \in U^{\mathscr{X}} \text {. }
$$

Then $C$ is in $\Gamma$, since $\Gamma$ is closed under intersections and recursive preimages (projections are recursive). So for some $e_{0}$

$$
\begin{aligned}
(m, n, x) \in C & \Leftrightarrow\left(e_{0}, m, n, x\right) \in U^{\omega^{2} \times \mathscr{Z}} \\
& \Leftrightarrow\left(S^{2, \mathscr{X}}\left(e_{0}, m, n\right), x\right) \in U^{\mathscr{Z}},
\end{aligned}
$$

so let $f(m, n)=S^{2, \mathscr{Z}}\left(e_{0}, m, n\right)$.

Similarly, for $\Gamma=\Sigma_{1}^{1}$ for example, one can compute effectively, given a code of a $\Sigma_{1}^{1}$ set $A \subseteq \mathscr{X} \times \mathscr{Y}$, a code of its projection onto $\mathscr{X}$, etc.

Although there are no universal sets for the class $\Delta_{1}^{1}$ (as an easy diagonalization argument shows, using the fact that $\Delta_{1}^{1}$ is closed under complements), one can still use the idea of coding for this class as well. If $A \subseteq \mathscr{X}$ is $\Delta_{1}^{1}$ then both $A$ and $\mathscr{X}-A$ are $\Sigma_{1}^{1}$ and any pair $(n, m)$, where $\left(U_{\Sigma_{1}^{1}}^{\mathscr{X}}\right)_{n}=A,\left(U_{\Sigma_{1}^{1}}^{\mathscr{X}}\right)_{m}=\mathscr{X}-A$, is called a code for $A$. With this notion of a code for $\Delta_{1}^{1}$ all the closure properties of $\Delta_{1}^{1}$ hold effectively in the codes as well.

But one can go beyond closure properties to other structural properties and establish corresponding effective uniformities. Take as a typical example the so-called reduction property for $\Pi_{1}^{1}$ sets: Given, $A, B \subseteq \mathscr{X}$ in $\Pi_{1}^{1}$ we can find $A^{*}, B^{*} \subseteq \mathscr{X}$ also in $\Pi_{1}^{1}$ reducing $A, B$, i.e., $A^{*} \subseteq A, B^{*} \subseteq B, A^{*} \cap$ $B^{*}=\varnothing, A^{*} \cup B^{*}=A \cup B$. (This is Theorem 7.3.1 in [MK].) We claim that we can effectively find codes of some such $A^{*}, B^{*}$, given codes of $A, B$. Indeed consider $U^{\mathscr{X}}$ and define $(m, n, x) \in U_{0} \Leftrightarrow(m, x) \in U^{\mathscr{X}},(m, n, x) \in$ $U_{1} \Leftrightarrow(n, x) \in V^{\mathscr{X}}$. Clearly $U_{0}, U_{1}$ are $\Pi_{1}^{1}$, so by the reduction property find $U_{0}^{*}, U_{1}^{*}$ in $\Pi_{1}^{1}$ reducing $U_{0}, U_{1}$. Then for some $e_{0}, e_{1}$

$$
\begin{aligned}
(m, n, x) \in U_{i}^{*} & \Leftrightarrow\left(e_{i}, m, n, x\right) \in U^{\omega^{2} \times \mathscr{Z}} \\
& \Leftrightarrow\left(S^{2, \mathscr{X}}\left(e_{i}, m, n\right), x\right) \in U^{\mathscr{L}} .
\end{aligned}
$$

Put $S^{2, \mathscr{X}}\left(e_{i}, m, n\right)=f_{i}(m, n)$.

If $A=U_{m}^{\mathscr{X}}, B=U_{n}^{\mathscr{X}}$, then $A^{*}=U_{f_{1}(m, n)}^{\mathscr{X}}, B^{*}=U_{f_{2}(m, n)}^{\mathscr{D}}$ reduce $A, B$. 
A corollary of the reduction property for $\Pi_{1}^{1}$ sets is the separation property for $\Sigma_{1}^{1}$ sets: Given disjoint $A, B \subseteq \mathscr{Z}$ in $\Sigma_{1}^{1}$ there is a $C$ in $\Delta_{1}^{1}$ with $A \subseteq C$, $B \cap C=\varnothing$. To find $C$ let $A^{\prime}=\mathscr{X}-A, B^{\prime}=\mathscr{X}-B$ and let $A^{*}, B^{*}$ in $\Pi_{1}^{1}$ reduce $A^{\prime}, B^{\prime}$. Then take $C=\mathscr{X}-A^{*}$ and notice that $\mathscr{X}-C=\mathscr{X}-B^{*}$. This argument shows that separation holds effectively in the codes: one can effectively find a code of such a $C$ given codes of $A, B$.

3.3. Another coding of the $\Delta_{1}^{1}$ sets. For some purposes the coding system we introduced in $\S 3.2$ for $\Delta_{1}^{1}$ sets is not the appropriate one. The basic problem is that the property of a pair $(m, n) \in \omega^{2}$ of being a code of a $\Delta_{1}^{1}$ set is too complicated, i.e., not $\Pi_{1}^{1}$. We can find a different coding system retaining the crucial properties of the original one but with a $\Pi_{1}^{1}$ set of codes. More precisely, we have

Theorem 3.3.1. Given a product space $\mathscr{X}$ there is a $\Pi_{1}^{1}$ set $C^{\mathscr{X}} \subseteq \omega$ and sets $P^{\mathscr{Z}}, S^{\mathscr{L}} \subseteq \omega \times \mathscr{X}$ in $\Pi_{1}^{1}, \Sigma_{1}^{1}$ resp. such that for $n \in C^{\mathscr{X}}$

$$
P_{n}^{\mathscr{X}}=S_{n}^{\mathscr{Z}}:=D_{n}^{\mathscr{X}}
$$

and for $A \subseteq \mathscr{X}, A \in \Delta_{1}^{1}$ iff $\exists n \in C^{\mathscr{X}} \quad\left(A=D_{n}^{\mathscr{X}}\right)$.

The $\Pi_{1}^{1}$ set $C^{\mathscr{X}}$ is the set of "nice codes" for $\Delta_{1}^{1}$ and if $A=D_{n}^{\mathscr{X}}$ we call $n$ a "nice code" for $A$. The sets $P^{\mathscr{X}}, S^{\mathscr{Z}}$ provide, uniformly in $n, \Pi_{1}^{1}, \Sigma_{1}^{1}$ definitions of $A$.

Proof. Let $m \mapsto\left((m)_{0},(m)_{1}\right)$ be a recursive bijection of $\omega$ with $\omega \times \omega$. Consider $U^{\mathscr{Q}}$ and define

$$
\begin{aligned}
& (n, x) \in U_{0} \Leftrightarrow\left((n)_{0}, x\right) \in U^{\mathscr{Z}}, \\
& (n, x) \in U_{1} \Leftrightarrow\left((n)_{1}, x\right) \in U^{\mathscr{Z}} .
\end{aligned}
$$

Then $U_{0}, U_{1}$ are $\Pi_{1}^{1}$, so reduce them to get $U_{0}^{*}, U_{1}^{*}$, also $\Pi_{1}^{1}$. Define now

$$
\begin{aligned}
C^{\mathscr{X}} & =\left\{n:\left(U_{0}^{*}\right)_{n} \cup\left(U_{1}^{*}\right)_{n}=\mathscr{X}\right\} \\
& =\left\{n: \forall x \in \mathscr{X}\left[(n, x) \in U_{0}^{*} \vee(n, x) \in U_{1}^{*}\right]\right\} .
\end{aligned}
$$

Clearly $C^{\mathscr{X}}$ is $\Pi_{1}^{1}$. Now let

$$
P^{\mathscr{X}}=U_{0}^{*}, \quad S^{\mathscr{X}}=(\omega \times \mathscr{X})-U_{1}^{*} .
$$

If $n \in C^{\mathscr{X}}$, then since $U_{0}^{*}, U_{1}^{*}$ are disjoint $\left(U_{0}^{*}\right)_{n},\left(U_{1}\right)_{n}^{*}$ partition $\mathscr{X}$, so $P_{n}^{\mathscr{X}}=S_{n}^{\mathscr{X}}:=D_{n}^{\mathscr{X}}$. Clearly if $n \in C^{\mathscr{X}}, D_{n}^{\mathscr{X}}$ is $\Delta_{1}^{1}$. Conversely if $A \subseteq \mathscr{X}$ is $\Delta_{1}^{1}$ let $k, l$ be such that $A=U_{k}^{\mathscr{X}}=\mathscr{X}-U_{l}^{\mathscr{X}}$. Let $n$ be such that $(n)_{0}=k,(n)_{1}=$ $l$. Then $\left(U_{0}\right)_{n}=A,\left(U_{1}\right)_{n}=\mathscr{X}-A$, so $\left(U_{0}^{*}\right)_{n}=A,\left(U_{1}^{*}\right)_{n}=\mathscr{X}-A$, thus $n \in C^{\mathscr{Z}}$ and $D_{n}^{\mathscr{X}}=A$. 
3.4. Relativization. Relationship of the effective and the classical notions. The basic notions of effective descriptive set theory can be relativized to an arbitrary parameter $x$ in $\mathscr{N}$. First one defines the concept of a function $f: \omega^{n} \rightarrow \omega$ being recursive in $x$. (Intuitively this means that there is an algorithm to compute $f$ which is allowed to ask occasionally for values $x(i)$ of $x$ which are understood to be supplied by an "oracle" for $x$, as generally $x$ itself is not computable. Clearly $x$ is recursive in $x$.) Once this has been done, it is straightforward to define the $\Sigma_{1}^{0}$ sets relativized to $x$, in symbols $\Sigma_{1}^{0}(x)$. For example for $\mathscr{X}=\omega \times \mathscr{N}$ these are the sets of the form $\bigcup_{n}\left\{i_{n}\right\} \times \mathscr{N}_{s_{n}}$, where $n \mapsto i_{n}, n \mapsto s_{n}$ are recursive in $x$. Then $\Pi_{1}^{0}(x)$ are the complements of $\Sigma_{1}^{0}(x)$ sets and $\Sigma_{1}^{1}(x)$ are the projections of $\Pi_{1}^{0}(x)$ sets, while $\Pi_{1}^{1}(x)$ sets are complements of $\Sigma_{1}^{1}(x)$ sets. Using $\Sigma_{1}^{0}(x)$ instead of $\Sigma_{1}^{0}$ one also defines the concept of a recursive in $x$ function between product spaces.

All the results of the effective theory we have discussed hold when relativized to an arbitrary parameter $x$, as can be seen immediately by just replacing in their proofs the notions $\Sigma_{1}^{0}, \Pi_{1}^{0}, \ldots$, recursive by $\Sigma_{1}^{0}(x), \Pi_{1}^{0}(x), \ldots$, recursive in $x$. (Ultimately of course this is based on the fact that the basic structural properties of computability theory carry over to their relativized versions.) For example the following relativized reduction property holds: Given $A, B \subseteq \mathscr{X}$ which are $\Pi_{1}^{1}(x)$, we can find $A^{*}, B^{*}$ in $\Pi_{1}^{1}(x)$ which reduce $A, B$.

The concept of relativization provides the bridge between the effective and the classical descriptive set theory. Denote by $\Sigma_{1}^{0}$ the class $\bigcup_{x \in \mathscr{N}} \Sigma_{1}^{0}(x)$ (i.e., $A$ is $\Sigma_{1}^{0}$ iff $\exists x \in \mathscr{N} \quad\left(A\right.$ is $\left.\left.\Sigma_{1}^{0}(x)\right)\right)$. Similarly let $\boldsymbol{\Pi}_{1}^{0}=\bigcup_{x \in \mathscr{N}} \Pi_{1}^{0}(x), \Sigma_{1}^{1}=$ $\bigcup_{x \in \mathscr{N}} \Sigma_{1}^{1}(x), \Pi_{1}^{1}=\bigcup_{x \in \mathscr{N}} \Pi_{1}^{1}(x), \Delta_{1}^{1}=\bigcup_{x \in \mathcal{N}} \Delta_{1}^{1}(x)=\Sigma_{1}^{1} \cap \Pi_{1}^{1}$. Then it is immediate that

$$
\begin{array}{ll}
\Sigma_{1}^{0}=\text { open }, & \Pi_{1}^{0}=\text { closed }, \\
\Sigma_{1}^{1}=\text { analytic }, & \Pi_{1}^{1}=\text { coanalytic } .
\end{array}
$$

For example, for the space $\mathscr{N}$, an arbitrary open set is of the form $U=\bigcup_{n} \mathscr{N}_{s_{n}}$ and if $x=\left(n \mapsto s_{n}\right)$, then clearly $U$ is $\Sigma_{1}^{0}(x)$. Similarly a function between product spaces is continuous iff it is recursive in some $x$.

Thus effective descriptive set theory is a refinement of classical descriptive set theory and effective results via relativization imply their classical versions. For example, consider again the reduction property. The classical version says that given coanalytic sets $A, B \subseteq \mathscr{X}$ there are coanalytic sets $A^{*}, B^{*}$ reducing $A, B$. Let $x \in \mathscr{N}$ be such that $A, B$ are simultaneously $\Pi_{1}^{1}(x)$ (such $x$ can be found since $(A \times\{0\}) \cup(B \times\{1\}) \subseteq \mathscr{X} \times \omega$ is $\Pi_{1}^{1}$, so $\Pi_{1}^{1}(x)$ for some $\left.x\right)$. The effective reduction theorem gives that the reduction property holds for $\Pi_{1}^{1}$ and thus by relativization for $\Pi_{1}^{1}(x)$, so we can find such $A^{*}, B^{*}$ which are $\Pi_{1}^{1}(x)$, in particular $\Pi_{1}^{1}$.

Our main theorem (in the case $X=\mathscr{N}$-the general case can be easily 
deduced from that) states that for any Borel equivalence relation $E$ on $\mathcal{N}$ either $E$ has a Borel separating family or else $E_{0} \sqsubseteq E$ (continuously). It will be therefore enough to prove the following effective version, recalling that by Souslin's Theorem, Borel = analytic-coanalytic $=\Delta_{1}^{1}:$ If $E$ is a $\Delta_{1}^{1}$ equivalence relation on $\mathscr{N}$, then either $E$ has a $\Delta_{1}^{1}$ separating family (i.e., a family $\left\{A_{n}\right\}$ with each $A_{n}$ in $\Delta_{1}^{1}$ ) or else $E_{0} \sqsubseteq E$ (continuously). By relativization this is going to hold for $E$ in $\Delta_{1}^{1}(x)$ (with a $\Delta_{1}^{1}(x)$ separating family in the first alternative) and thus for $E$ in $\Delta_{1}^{1}$ (with a $\Delta_{1}^{1}=$ Borel separating family in the first alternative).

\section{The GANDY-HARRINGTON TOPOLOGY}

We will discuss here the Gandy-Harrington topology on $\mathscr{N}$ (based on effective descriptive set theory), which will be the main tool for the proof of our result.

Definition 4.1. The Gandy-Harrington topology on $\mathcal{N}$, denoted by $\tau$, is the topology generated by the $\Sigma_{1}^{1}$ subsets of $\mathscr{N}$. Since $\Sigma_{1}^{1}$ is closed under finite intersections it follows that a set $U \subseteq \mathscr{N}$ is in $\tau$ iff $U=\cup_{n} U_{n}$, where $U_{n} \in \Sigma_{1}^{1}$

We also let $\tau_{n}$ be the topology on $\mathscr{N}^{n}$ generated by the $\Sigma_{1}^{1}$ subsets of $\mathscr{N}^{n}$. Since $\mathscr{N}^{n}$ and $\mathscr{N}$ are recursively homeomorphic, $\left(\mathscr{N}^{n}, \tau_{n}\right)$ is homeomorphic to $(\mathscr{N}, \tau) \quad\left(\tau=\tau_{1}\right)$. Clearly every basic open set in the standard topology of $\mathscr{N}^{n}$ is $\Sigma_{1}^{1}$, so $\tau_{n}$ extends the standard topology. In particular, $\tau_{n}$ is Hausdorff. Also $\tau_{n}$ has a countable basis.

Although $\tau$ does not have some "nice" properties desirable in a topology, e.g., is not regular (this is because there are $\Sigma_{1}^{1}$, so open in $\tau$ sets which are not $\Pi_{1}^{1}$, and thus not $F_{\sigma}$ in $\tau$ ), it satisfies one crucial property that allows the application of category arguments.

Theorem 4.2. The space $\left(\mathscr{N}^{n}, \tau_{n}\right)$ is a strong Choquet space.

Proof. It is enough of course to work with $n=1$. So consider $(\mathscr{N}, \tau)$.

Recall from $\S 3$ that every $\Sigma_{1}^{l}$ subset of $\mathscr{N}$ is of the form $p[T]$, for some recursive tree $T$ on $\omega \times \omega$ (and vice versa). We will describe now a winning strategy for $\alpha$ in the strong Choquet game for $(\mathcal{N}, \tau)$.

Let $\beta$ start by playing $\left(x_{0}, U_{0}\right)$. Let $A_{0} \in \Sigma_{1}^{1}$ be such that $x_{0} \in A_{0} \subseteq U_{0}$ and let $T_{0}$ be a recursive tree with $A_{0}=p\left[T_{0}\right]$. As $x_{0} \in p\left[T_{0}\right]$ find $y_{0}$ (a "witness") such that $\left(x_{0}, y_{0}\right) \in\left[T_{0}\right]$. For any tree $T$ on $\omega \times \omega$ and any $(s, t) \in T$ let $T_{(s, t)}=\left\{\left(s^{\prime}, t^{\prime}\right):\left(s^{\prime} \subseteq s\right.\right.$ or $\left.s^{\prime} \supseteq s\right)$ and $\left(t^{\prime} \subseteq t\right.$ or $\left.t^{\prime} \supseteq t\right)$ and $\left.\left(s^{\prime}, t^{\prime}\right) \in T\right\}$. If $T$ is recursive, so is $T_{(s, t)}$. Now let $s_{0}=x_{0} \uparrow 1, t_{0}^{0}=y_{0} \uparrow 1$, where $x \uparrow n=(x(0), \ldots, x(n-1))$. If $V_{0}=p\left[\left(T_{0}\right)_{\left(s_{0}, t_{0}^{0}\right)}\right]$, then $V_{0} \in \Sigma_{1}^{1}$, $V_{0} \subseteq A_{0} \subseteq U_{0}$, and $x_{0} \in V_{0}$. Let $\alpha$ play this $V_{0}$ in his first move. 
Let $\beta$ next play $\left(x_{1}, U_{1}\right), U_{1} \subseteq V_{0}$. As $x_{i} \in V_{0}$ there is a witness $y_{0}^{\prime}$ with $\left(x_{1}, y_{0}^{\prime}\right) \in\left[\left(T_{0}\right)_{\left(s_{0}, t_{0}^{0}\right)}\right]$ so if $s_{1}=x_{1} \backslash 2, t_{1}^{0}=y_{0}^{\prime} \backslash 2$, then $s_{0} \subseteq s_{1}, t_{0}^{0} \subseteq t_{1}^{0}$. Also fix $A_{1} \in \Sigma_{1}^{1}$ with $x_{1} \in A_{1} \subseteq U_{1}$ and $T_{1}$ recursive with $A_{1}=p\left[T_{1}\right]$. Then let $y_{1}$ be a witness with $\left(x_{1}, y_{1}\right) \in\left[T_{1}\right]$. Let $t_{0}^{1}=y_{1} \mid 1$. If $V_{1}=$ $p\left[\left(T_{0}\right)_{\left(s_{1}, t_{1}^{0}\right)}\right] \cap p\left[\left(T_{1}\right)_{\left(s_{0}, t_{0}^{1}\right)}\right]$, then $V_{1} \in \Sigma_{1}^{1}, V_{1} \subseteq A_{1} \subseteq U_{1}$, and $x_{1} \in V_{1}$. This $V_{1}$ is $\alpha$ 's answer to $\left(x_{1}, U_{1}\right)$.

Proceeding this way, when $\beta$ plays $\left(x_{0}, U_{0}\right),\left(x_{1}, U_{1}\right), \ldots \alpha$ produces $V_{0}, V_{1}, \ldots$ with $U_{0} \supseteq V_{0} \supseteq U_{1} \supseteq V_{1} \supseteq \cdots, x_{n} \in V_{n}$, and moreover one defines for each $n$ a recursive tree $T_{n}$ with $x_{n} \in A_{n}=p\left[T_{n}\right] \subseteq U_{n}$ and sequences $s_{0} \subseteq$ $s_{1} \subseteq \cdots, t_{0}^{n} \subseteq t_{1}^{n} \subseteq t_{2}^{n} \subseteq \cdots$ with $\left(s_{k}, t_{k}^{n}\right) \in T_{n}$, such that for each $k, s_{k}, t_{k}^{n}$ have length $k+1$ and $V_{k}=p\left[\left(T_{0}\right)_{\left(s_{k}, t_{k}^{0}\right)}\right] \cap p\left[\left(T_{1}\right)_{\left(s_{k-1}, t_{k-1}^{1}\right)}\right] \cap \cdots \cap p\left[\left(T_{k}\right)_{\left(s_{0}, t_{0}^{k}\right)}\right]$. Let $x=\bigcup_{k} s_{k} \in \mathscr{N}$. We will show that $x \in \bigcap_{n} A_{n} \subseteq \cap V_{n}$. Consider $A_{n}=p\left[T_{n}\right]$. Let $y_{n}=\bigcup_{k} t_{k}^{n}$. As $\left(s_{k}, t_{k}^{n}\right) \in T_{n}$, clearly $\left(x, y_{n}\right) \in\left[T_{n}\right]$, so $x \in p\left[T_{n}\right]$.

Consider now the space $\mathscr{N}^{2}$. It carries the two topologies $\tau_{2}$ and $\tau \times \tau$ (i.e., the product topology of $\tau$ with itself). As every rectangle $A \times B$ with $A, B \in \Sigma_{1}^{1}$ is also $\Sigma_{1}^{1}$, clearly $\tau \times \tau \subseteq \tau_{2}$ but $\tau_{2} \neq \tau \times \tau$ (since $\{(x, y): x=y\}$ is in $\tau_{2}$ but not in $\tau \times \tau)$. There is however a simple but important fact which shows that $\tau_{2}$ retains some of the properties of $\tau \times \tau$, namely that the projection map $\pi(x, y)=x$ is open. (It is obviously continuous as $\tau \times \tau \subseteq \tau_{2}$.) We will formulate this in more general forms convenient for our applications.

Let $n \geq n^{\prime}$. A projection of $\mathscr{N}^{n}$ onto $\mathscr{N}^{n^{\prime}}$ is any map of the form $\pi\left(x_{1}, \ldots, x_{n}\right)=\left(x_{i_{1}}, \ldots, x_{i_{n^{\prime}}}\right)$, where $1 \leq i_{1}<\cdots<i_{n^{\prime}} \leq n$. A projection of $\mathscr{N}^{n} \times \mathscr{N}^{m}=\mathscr{N}^{n+m}$ onto $\mathscr{N}^{n^{\prime}} \times \mathscr{N}^{m^{\prime}}=\mathscr{N}^{n^{\prime}+m^{\prime}}$, where $n \geq n^{\prime}, m \geq m^{\prime}$, is any map of the form $\pi\left(x_{1}, \ldots, x_{n}, y_{1} \cdots y_{m}\right)=\left(\pi_{1}\left(x_{1} \cdots x_{n}\right), \pi_{2}\left(y_{1} \cdots y_{m}\right)\right)$, where $\pi_{1}, \pi_{2}$ are projections of $\mathscr{N}^{n}$ onto $\mathscr{N}^{n^{\prime}}, \mathscr{N}^{m}$ onto $\mathscr{N}^{m^{\prime}}$ resp.

Proposition 4.3. Let $n \geq n^{\prime}, m \geq m^{\prime}$. Any projection of $\left(\mathscr{N}^{n} \times \mathscr{N}^{m}, \tau_{n} \times \tau_{m}\right)$ onto $\left(\mathscr{N}^{n^{\prime}} \times \mathscr{N}^{m^{\prime}}, \tau_{n^{\prime}} \times \tau_{m^{\prime}}\right)$ is open (and obviously continuous).

Proof. Take for notational simplicity $n=m=2, n^{\prime}=m^{\prime}=1$ and consider the projection $\pi(x, y, z, t)=(x, z)$. Let $G$ be open in $\left(\mathscr{N}^{2} \times \mathscr{N}^{2}, \tau_{2} \times \tau_{2}\right)$. Then $G=\bigcup_{n}\left(U_{n} \times V_{n}\right)$, where $U_{n}, V_{n}$ are open in $\tau_{2}$, i.e., of the form $U_{n}=$ $\bigcup_{k} U_{n, k}, V_{n}=\bigcup_{l} V_{n, l}$ with $U_{n, k}, V_{n, l} \Sigma_{1}^{1}$ subsets of $\mathscr{N}^{2}$. Then $\pi(G)=$ $\bigcup_{n} \pi\left(U_{n} \times V_{n}\right)=\bigcup_{n} \bigcup_{k} \bigcup_{l} \pi\left(U_{n, k} \times V_{n, l}\right)=\bigcup_{n} \bigcup_{k} \bigcup_{l}\left(A_{n, k} \times B_{n, l}\right)$, where $x \in$ $A_{n, k} \Leftrightarrow \exists y(x, y) \in U_{n, k}$, so that $A_{n, k}$ is $\Sigma_{1}^{1}$, and similarly for $B_{n, l}$. So clearly $\pi(G)$ is open in $\tau \times \tau$.

We will actually need a further strengthening of this proposition in the next section. Let $R$ be a $\Sigma_{1}^{1}$ equivalence relation on $\mathscr{N}$ (i.e., $R \subseteq \mathscr{N}^{2}$ is $\Sigma_{1}^{1}$ ). For $n \geq 2$ let $R^{n}=\left\{\left(x_{1} \cdots x_{n}\right) \in \mathscr{N}^{n}: x_{i} R x_{j} \forall i, j\right\}$. For any $n, m$ denote 
by $\left(R^{n+m}, \tau_{n} \times \tau_{m}\right)$ the space $R^{n+m} \subseteq \mathscr{N}^{n} \times \mathscr{N}^{m}$ with the relative topology it inherits from $\left(\mathscr{N}^{n+m}, \tau_{n} \times \tau_{m}\right)$. If $n^{\prime} \leq n, m^{\prime} \leq m$ and $\pi$ is any projection of $\mathscr{N}^{n} \times \mathscr{N}^{m}$ onto $\mathscr{N}^{n^{\prime}} \times \mathscr{N}^{m^{\prime}}$ then clearly $\pi$ maps $R^{n+m}$ onto $R^{n^{\prime}+m^{\prime}}$.

Proposition 4.4. Let $R$ be a $\Sigma_{1}^{1}$ equivalence relation on $\mathscr{N}$ and $n \geq n^{\prime}, m \geq$ $m^{\prime}$. Let $\tau$ be a projection of $\mathscr{N}^{n} \times \mathscr{N}^{m}$ onto $\mathscr{N}^{n^{\prime}} \times \mathcal{N}^{m^{\prime}}$. Then $\pi$ is an open (and continuous) map from $\left(R^{n+m}, \tau_{n} \times \tau_{m}\right)$ onto $\left(R^{n^{\prime}+m^{\prime}}, \tau_{n^{\prime}} \times \tau_{m^{\prime}}\right)$.

Proof. Take for notational simplicity $n=m=2, n^{\prime}=m^{\prime}=1, \pi(x, y, z, t)=$ $(x, z)$. Let $U, V$ be $\Sigma_{1}^{1}$ subsets of $\mathscr{N}^{2}$, so that $(U \times V) \cap R^{4}$ is a basic open set in $\left(R^{4}, \tau_{2} \times \tau_{2}\right)$. Let $U^{\prime}=\{x: \exists y[(x, y) \in U$ and $x R y]\}, V^{\prime}=\{z$ : $\exists t[(z, t) \in U$ and $z R t]\}$. As $R$ is $\Sigma_{1}^{1}, U^{\prime}, V^{\prime}$ are $\Sigma_{1}^{1}$ too. Now, easily, using the fact that $R$ is an equivalence relation, $\left(U^{\prime} \times V^{\prime}\right) \cap R=\pi\left((U \times V) \cap R^{4}\right)$, so that $\pi\left((U \times V) \cap R^{4}\right)$ is open in $R\left(=R^{2}\right)$ and we are done.

Finally recall for further reference the following obvious facts from topology: If $F: X \rightarrow Y$ is open and continuous, then the inverse image of a dense set in $Y$ is dense in $X$ and the inverse image of a comeager set in $Y$ is comeager in $X$. (A set is comeager if it contains the intersection of a countable sequence of dence open sets.) Moreover, if $G$ is open in $Y$ and $B \subseteq Y$ is comeager in $G$ (i.e., $B \cap G$ contains the intersection of a countable sequence of open, dense in $G$ sets), then $f^{-1}[B]$ is comeager in the open set $f^{-1}[G]$.

\section{Proof of THE MAIN THEOREM}

Let us first show how Theorem 1.1 and its consequences 1.2 and 1.3 follow from Theorem 1.4. We will then concentrate on the hard part, the proof of 1.4.

Proof of 1.1, 1.2, and 1.3 from 1.4. By direct relativization arguments, as discussed in $\S 3.4$, Theorem 1.4 implies the dichotomy (I) $E$ is smooth or (II) $E_{0} \sqsubseteq E$ (continuously) for any Borel equivalence relation $E$ on the space $\mathscr{N}$. To get it for an arbitrary Polish space $X$, or even for an arbitrary Borel set $B \subseteq X$, one uses the following classical transfer theorem: Every Borel set $B$ in a Polish space $X$ is the image of a closed subset $F$ of $\mathscr{N}$ by a continuous 1-1 mapping $f: F \rightarrow X$. So suppose $E$ is a Borel equivalence relation on $B$, and define $E^{*}$ on $\mathcal{N}$ by $x E^{*} y \Leftrightarrow(x, y \notin F$ and $x=y)$ or $(x \in F$ and $y \in F$ and $f(x) E f(y))$. Clearly $E^{*}$ is Borel. If (I) holds for $E^{*}$, and $\left\{A_{n}^{*}\right\}$ is a Borel separating family for it, the family $A_{n}=f\left[F \cap A_{n}^{*}\right]$ is clearly a Borel separating family for $E$. If now (II) holds for $E^{*}$, and $\varphi: 2^{\omega} \rightarrow \mathscr{N}$ is a continuous $1-1$ function with $\alpha E_{0} \beta \Leftrightarrow \varphi(\alpha) E^{*} \varphi(\beta)$, note that because of the cardinality of each $E_{0}$-class, one must have $\varphi\left[2^{\omega}\right] \subseteq F$. But then $f \circ \varphi$ is a continuous $1-1$ embedding of $2^{\omega}$ into $B$ with $\alpha E_{0} \beta \Leftrightarrow f \circ \varphi(\alpha) E f \circ \varphi(\beta)$ and (II) holds for E.

We now prove the rest of the assertions of 1.1. First if $E$ had a separating family of universally measurable sets, and $E_{0}$ is reducible to $E$ via a universally 
measurable function, so would $E_{0}$, a contradiction. If (I)* holds, i.e., $E$ has a $C$-measurable selector $s$, then the family $s^{-1}\left[U_{n}\right]$, for $U_{n}$ a basis of the topology of $X$, forms a $C$-measurable separating family, and hence (I) follows. To show $(\mathrm{I}) \Rightarrow(\mathrm{I})^{*}$, let $\left\{A_{n}\right\}$ be a Borel separating family, and define $\varphi: X \rightarrow$ $2^{\omega}$ by

$$
\varphi(x)(n)= \begin{cases}1 & \text { if } x \in A_{n} \\ 0 & \text { otherwise }\end{cases}
$$

Then $\varphi$ is Borel and $\varphi(x)=\varphi(y) \Leftrightarrow x E y$. Let $Y=\varphi[X]$, and consider the Borel set $A=\{(y, x) \in Y \times X: y=\varphi(x)\}$. By a rlassical uniformization result, there is a $C$-measurable selector of $A$, i.e., a function $\Psi: Y \rightarrow X$ with $(y, \Psi(y)) \in A$ for all $y \in Y$. But then $s=\Psi \circ \varphi$ is a $C$-measurable selector for $E$.

To prove $(\mathbf{I}) \Rightarrow(\mathbf{I})^{* *}$, note that given any countable collection of Borel subsets of $X$, one can define a Polish extension of the topology of $X$ for which these sets are clopen. Applying this to a Borel separating family for $E$ gives a Polish extension $\sigma$ of the topology of $X$ for which $E$ is $(\sigma \times \sigma)$-closed.

For $(\mathrm{I} I)^{* *} \Rightarrow(\mathrm{I})$, it is enough to prove the classical fact that any $G_{\delta}$ equivalence relation on a Polish space has a $\Sigma_{1}^{1}$ separating family. To see this, note that if $x$ and $y$ are not $E$-equivalent, $[x]_{E}$ and $[y]_{E}$ are two disjoint $G_{\delta}$ 's, so by the Baire category theorem, their closures must be distinct. So the family $\left[U_{n}\right]_{E}=\left\{x: \exists y \in U_{n}(x E y)\right\}$, where $U_{n}$ varies over a basis of the topology of $X$, is a $\Sigma_{1}^{1}$ separating family.

For (II) $\Rightarrow(\text { II })^{*}$, let $\lambda$ be the standard Lebesgue measure on $2^{\omega}$; if $E_{0} \leq$ $E$ via $f$, let $\mu=f \lambda$. One immediately checks that $\mu$ is (E-)ergodic and nonatomic. For the converse, recall that the existence of an ( $E$-)ergodic, nonatomic measure implies that $E$ is not smooth.

For Corollary 1.2, we already noticed that a $G_{\delta}$ equivalence relation always has a $\Sigma_{1}^{1}$ separating family. Using Theorem 1.1 we get the result.

For Corollary 1.3, we already noticed that given any Borel $B \subseteq X$, the dichotomy (I) or (II) holds for the restriction $E \uparrow B$ of $E$ to $B$. So if $B$ is not $E$-smooth, one can embed $E_{0}$ into $E \uparrow B$ and as before, this gives an ergodic nonatomic probability measure $\mu$ for $E \backslash B$, hence for $E$, with $\mu(B)=1$. Conversely if $\mu(B)>0$ for some ergodic nonatomic measure $\mu$ for $E$, then $\mu \nmid B$ is an ergodic nonatomic measure for $E \uparrow B$, and $B$ cannot be $E$-smooth.

Proof of 1.4. From now on, $E$ is a fixed $\Delta_{1}^{1}$ equivalence relation on $\mathscr{N}$. For a subset $A$ of $\mathscr{N}$, we denote by $[A]_{E}$ its $E$-saturation, i.e., $[A]_{E}=\{x: \exists y \in$ $A(x E y)\}$. We call $A$ E-invariant if $A=[A]_{E}$.

Lemma 5.1. If $A, B$ are two $\Sigma_{1}^{1}$ sets and $[A]_{E} \cap[B]_{E}=\varnothing$, there exists a $\Delta_{1}^{1}$ $E$-invariant set $C$ with $A \subseteq C$ and $B \cap C=\varnothing$.

Proof. The idea of the proof is the following: Clearly for any $\Sigma_{1}^{1}$ set $A,[A]_{E}=$ $\{x: \exists y \in A(x E y)\}$ is $\Sigma_{1}^{1}$ by the closure properties of $\Sigma_{1}^{1}$ sets. Now if $A, B$ 
are $\Sigma_{1}^{1}$ and $[A]_{E} \cap[B]_{E}=\varnothing$, we can find by the separation theorem a $\Delta_{1}^{1}$ set $C_{0}$ with $[A]_{E} \subseteq C_{0}$ and $C_{0} \cap[B]_{E}=\varnothing$. Now $\left[C_{0}\right]_{E}$ is $\Sigma_{1}^{1}$, and $\left[C_{0}\right]_{E} \cap[B]_{E}=$ $\varnothing$, hence we can find a $\Delta_{1}^{1}$ set $C_{1}$ with $\left[C_{0}\right]_{E} \subseteq C_{1}$ and $C_{1} \cap[B]_{E}=\varnothing$. Continuing this way, we get a sequence of $\Delta_{1}^{1}$ sets $C_{n}$ with $\left[C_{n}\right]_{E} \subseteq C_{n+1}$ and $C_{n+1} \cap[B]_{E}=\varnothing$. The set $C=\bigcup_{n} C_{n}=\bigcup_{n}\left[C_{n}\right]_{E}$ is $E$-invariant, and $A \subseteq C$, $C \cap B=\varnothing$. However the class $\Delta_{1}^{1}$ is not closed under countable unions, so to get $C$ in $\Delta_{1}^{1}$, one has to define the preceding sequence uniformly. Let $\left\{U_{n}^{\mathcal{N}}\right\}=\left\{U_{n}\right\}$ be a good universal system for $\Sigma_{1}^{1}$ subsets of $\mathcal{N}$, as discussed in $\S 3.2$. Recall that the separation theorem is uniform, i.e., there are recursive functions $f: \omega^{2} \rightarrow \omega$ and $g: \omega^{2} \rightarrow \omega$ such that if $U_{n}$ and $U_{m}$ are disjoint, then $U_{f(n, m)}=X-U_{g(n, m)}$ is a $\Delta_{1}^{1}$ set which separates them. Also, Theorem 3.2.1 implies easily that the map $A \mapsto[A]_{E}$ is uniform, for $\Sigma_{1}^{1}$ sets $A$. To see this, consider the set

$$
B=\left\{(n, x): \exists y \in U_{n}(x E y)\right\} .
$$

This set is $\Sigma_{1}^{1}$ in $\omega \times \mathscr{N}$, hence for some $e_{0}$

$$
\begin{aligned}
(n, x) \in B & \Leftrightarrow\left(e_{0}, n, x\right) \in U^{\omega \times \mathscr{N}} \\
& \Leftrightarrow\left(S^{1, \mathscr{N}}\left(e_{0}, n\right), x\right) \in U^{\mathscr{N}} .
\end{aligned}
$$

So the recursive function $h(n)=S^{1, \mathcal{N}}\left(e_{0}, n\right)$ satisfies $U_{h(n)}=\left[U_{n}\right]_{E}$.

Fix now a $\Sigma_{1}^{1}$ code $p_{0}$ for $[B]_{E}$, and pick $C_{0} \Delta_{1}^{1}$ with $[A]_{E} \subseteq C_{0}, C_{0} \cap$ $[B]_{E}=\varnothing$, and $\Sigma_{1}^{1}$ codes $n_{0}$ and $m_{0}$ for $C_{0}$ and $\mathcal{N}-C_{0}$ respectively. Define a function $k: \omega \rightarrow \omega$ recursively by

Also let

$$
\left\{\begin{array}{l}
k(0)=n_{0}, \\
k(n+1)=f\left(h(k(n)), p_{0}\right) .
\end{array}\right.
$$

$$
k^{\prime}(0)=m_{0}, \quad k^{\prime}(n+1)=g\left(h(k(n)), p_{0}\right) .
$$

Then clearly $k(n), k^{\prime}(n)$ are $\Sigma_{1}^{1}$ codes of $C_{n}$ and $\mathscr{N}-C_{n}$, for a sequence $\left\{C_{n}\right\}$ as above. And if we let $C=\bigcup_{n} C_{n}$, we get

$$
\begin{aligned}
x \in C & \Leftrightarrow \exists n\left(x \in U_{k(n)}\right) \\
& \Leftrightarrow \forall n\left(x \notin U_{k^{\prime}(n)}\right),
\end{aligned}
$$

so the $C$ is both $\Sigma_{1}^{1}$ and $\Pi_{1}^{1}$, hence $\Delta_{1}^{1}$ as desired.

Recall that $\tau$ denotes the Gandy-Harrington topology of $\mathscr{N}$. We define a new relation $\bar{E}$ on $\mathscr{N} \times \mathscr{N}$ as the closure of $E$ in the $\tau \times \tau$ topology.

Lemma 5.2. $\bar{E}$ is a $\Sigma_{1}^{1}$ equivalence relation which admits a separating family consisting of $\Delta_{1}^{1}$ sets, and hence is $G_{\delta}$ in $\tau \times \tau$.

Proof. By the definition of $\tau \times \tau$, if $(x, y) \notin \bar{E}$, there are two $\Sigma_{1}^{1}$ sets $A$ and $B$ with $x \in A, y \in B$, and $(A \times B) \cap E=\varnothing$, so that $[A]_{E} \cap[B]_{E}=\varnothing$. By 
5.1, there is $\Delta_{1}^{1} E$-invariant set $C$ with $x \in C$ and $y \notin C$. This means that

$$
\bar{E}=\bigcap\left\{(C \times C) \cup(\mathscr{N}-C) \times(\mathscr{N}-C): C \text { a } \Delta_{1}^{1} E \text {-invariant set }\right\} .
$$

So $\bar{E}$ is an equivalence relation, and the $\Delta_{1}^{1} E$-invariant sets form a separating family for it. As for each $\Delta_{1}^{1}$ set $C,(C \times C) \cup(\mathscr{N}-C) \times(\mathscr{N}-C)$ is clopen in $\tau \times \tau, \bar{E}$ is $G_{\delta}$. (It is of course also closed, but remember $\tau \times \tau$ is not regular, so closed does not imply $G_{\delta}$.) It remains to show that $\bar{E}$ is $\Sigma_{1}^{1}$. For this, we use the nice coding of $\Delta_{1}^{1}$ sets discussed in $\S 3.3$ : There is a $\Pi_{1}^{1}$ set $C \subseteq \omega$ and sets $P, S \subseteq \omega \times \mathscr{N}$ in $\Pi_{1}^{1}, \Sigma_{1}^{1}$ resp., such that for $n \in C, P_{n}=S_{n}$, and such that for $A \subseteq \mathscr{N}$

Using this, we can compute

$$
A \text { is } \Delta_{1}^{1} \Leftrightarrow \exists n \in C\left(A=P_{n}\right) \text {. }
$$

$$
\begin{aligned}
&(x, y) \notin \bar{E} \Leftrightarrow \exists A \in \Delta_{1}^{1}(A \text { is } E \text {-invariant and }(x \in A \wedge y \notin A)) \\
& \Leftrightarrow \exists n \in C\left(P_{n} \text { is } E \text {-invariant and }\left(x \in P_{n} \wedge y \notin S_{n}\right)\right) \\
& \Leftrightarrow \exists n \in C\left(\forall z \forall t\left(z \in S_{n} \wedge z E t \rightarrow t \in P_{n}\right)\right. \\
&\text { and } \left.\left(x \in P_{n} \wedge y \notin S_{n}\right)\right) .
\end{aligned}
$$

By the closure properties of $\Pi_{1}^{1}$, one gets that $(\mathscr{N} \times \mathscr{N})-\bar{E}$ is $\Pi_{1}^{1}$, so $\bar{E}$ is $\Sigma_{1}^{1}$.

It follows from Lemma 5.2 that if $\bar{E}=E$ (i.e., $E$ is $(\tau \times \tau)$-closed), then $E$ admits a separating family consisting of $\Delta_{1}^{1}$ sets, and we have case (I) of the dichotomy.

Although it is not useful for Theorem 1.1, let us show that in fact in this case one can find a separating family uniformly $\Delta_{1}^{1}$. To do this, one uses the following uniformization result (see [MK, 7.3.4]): If $A \subseteq \mathscr{X} \times \omega$ is $\Pi_{1}^{1}$, and if $\forall x \in X \exists n(x, n) \in A$, then there is a $\Delta_{1}^{1}$ function $F: \mathscr{X} \rightarrow \omega$ such that $\forall x(x, F(x)) \in A$. Apply this result to $\mathscr{X}=\mathscr{N} \times \mathscr{N}$ and the following set:

$$
\begin{aligned}
A=\left\{(x, y, n):(x, y) \in E \vee\left[(x, y) \notin E \wedge n \in C \wedge P_{n}\right.\right. & \text { is } E \text {-invariant } \\
& \left.\left.\left.\wedge x \in P_{n} \wedge y \notin S_{n}\right)\right]\right\} .
\end{aligned}
$$

By the same calculation as above, $A$ is $\Pi_{1}^{1}$. And if $E=\bar{E}$, we know that $\forall(x, y) \in \mathscr{N} \times \mathscr{N} \exists n(x, y, n) \in A$. So we can find $F \Delta_{1}^{1}$ with $(x, y, F(x, y))$ $\in A$ for all $(x, y) \in \mathscr{N} \times \mathscr{N}$. Define $D_{0}=F\left(\mathscr{N}^{2}-E\right)$. Then $D_{0}$ is a $\Sigma_{1}^{1}$ subset of $\omega$, and is contained in the $\Pi_{1}^{1}$ set $D_{1}=\left\{n \in C: P_{n}\right.$ is $E$-invariant $\}$. So by the separation theorem, there is a $\Delta_{1}^{1}$ set $D$ with $D_{0} \subseteq D \subseteq D_{1}$. Define now a sequence $\left\{A_{n}\right\}$ by $A_{n}=\mathscr{N}$ if $n \notin D, A_{n}=P_{n}$ if $n \in D$. As $D \subseteq D_{1}$, each $D_{n}$ is an $E$-invariant $\Delta_{1}^{1}$ set, and as $D_{0} \subseteq D$, the family $\left\{A_{n}\right\}_{n \in \omega}$ is a separating family for $E$. Finally

$$
\begin{aligned}
x \in A_{n} & \Leftrightarrow n \notin D \vee x \in P_{n} \\
& \Leftrightarrow n \notin D \vee x \in S_{n}
\end{aligned}
$$

is both $\Sigma_{1}^{1}$ and $\Pi_{1}^{1}$, so is $\Delta_{1}^{1}$. 
So from now on, we assume that $E \neq \bar{E}$, toward building a continuous embedding of $E_{0}$ into $E$.

Consider then the set

$$
X=\left\{x: E_{x} \neq \bar{E}_{x}\right\} .
$$

As $E \neq \bar{E}, X$ is not empty. Now by Lemma $5.2, X$ is $\Sigma_{1}^{1}$, as

$$
x \in X \leftrightarrow \exists y(x \bar{E} y \wedge x \not \dot{y})
$$

and $\bar{E}$ is $\Sigma_{1}^{1}$. Clearly, $X$ is also $\bar{E}$-invariant.

Lemma 5.3. Consider $\bar{E}$ with the (relative) $(\tau \times \tau)$-topology. Then $E$ is both dense and meager in the open set $(X \times X) \cap \bar{E}$.

Proof. Density is obvious. As $E$ is Borel for the usual topology, it is also Borel for $\tau \times \tau$, hence has the Baire property in $(\bar{E}, \tau \times \tau)$. So if $E$ is not meager in $(X \times X) \cap \bar{E}$, there must be two $\Sigma_{1}^{1}$ sets $A$ and $B, A, B \subseteq X$, with $(A \times B) \cap \bar{E} \neq \varnothing$ and $E$ comeager in $(A \times B) \cap \bar{E}$. By considering if necessary the two projections of $(A \times B) \cap \bar{E}$, we may assume that $\forall x \in A \exists y \in B(x \bar{E} y)$ and $\forall x \in B \exists y \in A(x \bar{E} y)$.

Claim. $A^{2} \cap \bar{E} \subseteq E$.

Granting this claim, we can finish the proof of the lemma as follows. First note that $[A]_{E}^{2} \cap \bar{E} \subseteq E$. (For if $x, y \in[A]_{E}$ and $x \bar{E} y$, and if $z, t \in A$ are such that $z E x$ and $y E t$, then $z \bar{E} t$, so $z E t$ as $A^{2} \cap \bar{E} \subseteq E$. But then $x E y$ by transitivity of $E$.) But this implies that in fact $[A]_{E}=[A]_{\bar{E}}$. Otherwise the set $A^{\prime}=\left\{x: \exists y \in[A]_{E}\left(x \bar{E} y \wedge x \not E^{\prime} y\right)\right\}$ is $\Sigma_{1}^{1}$ non- $\varnothing$, and $\left(A^{\prime} \times[A]_{E}\right) \cap \bar{E} \neq \varnothing$. By the density of $E$ in $\tau \times \tau,\left(A^{\prime} \times[A]_{E}\right) \cap E \neq \varnothing$. So there are $x \in A^{\prime}$ and $z \in[A]_{E}$ with $x E z$. And as $x \in A^{\prime}$, there is $y \in[A]_{E}$ with $x \bar{E} y$ and $x \not E y$. But then $y, z \in[A]_{E}$ and $y \bar{E} z$, so $y E z$ contradicting the transitivity of $E$.

Now if $x \in A$ and $y \bar{E} x$, then $y \in[A]_{E}$, and as $[A]_{E}^{2} \cap \bar{E} \subseteq E$, in fact $y E x$. This means $E_{x}=\bar{E}_{x}$, contradicting the fact that $A \subseteq X$.

It remains to prove the above claim that $A^{2} \cap \bar{E} \subseteq E$. Again this is done by contradiction. Consider the space $\bar{E}^{3}=\{(x, y, z): x \bar{E} y \bar{E} z\}$ with the induced $\left(\tau_{2} \times \tau\right)$-topology. It is a $G_{\delta}$-set in $\left(\mathscr{N}^{3}, \tau_{2} \times \tau\right)$ by Lemma 5.2 , hence a strong Choquet space, and in particular a Baire space by 4.2 and 2.1. Let $Y=\left\{(x, y, z) \in \bar{E}^{3}: x \in A \wedge y \in A \wedge z \in B\right\}$. This is an open nonempty set in $\left(\bar{E}^{3}, \tau_{2} \times \tau\right)$. The projections $(x, y, z) \mapsto(x, z)$ and $(x, y, z) \mapsto(y, z)$ are open: $\left(\bar{E}^{3}, \tau_{2} \times \tau\right) \rightarrow\left(\bar{E}^{2}, \tau \times \tau\right)$ by 4.4 , so as $E$ is comeager in $\bar{E} \cap(A \times B)$ for $\tau \times \tau$, the sets $Z_{1}=\{(x, y, z) \in Y: x E z\}$ and $Z_{2}=\{(x, y, z) \in Y: y E z\}$ are comeager in $Y$ for $\tau_{2} \times \tau$. But then, if $A^{2} \cap \bar{E} \nsubseteq E$, the $\Sigma_{1}^{1}$ set $Z_{3}=$ $\{(x, y, z) \in Y: x \not t y\}$ is a nonempty $\left(\tau_{2} \times \tau\right)$-open set, which by the Baire category theorem must meet $Z_{1} \cap Z_{2}$, so that we get $x, y, z$ with $x E z, y E z$ and $x \notin y$, a contradiction which proves the claim and finishes the proof of the lemma. 
By Lemma 5.3, we can find a decreasing sequence $\left\{W_{n}\right\}$ of $(\tau \times \tau)$-open sets with $W_{n} \subseteq X \times X, W_{n}$ dense in $(X \times X) \cap \bar{E}$, and $\bigcap_{n} W_{n} \cap E=\varnothing$. Moreover, as the diagonal $\Delta$ is contained in $E$ and is $(\tau \times \tau)$-closed, we may assume $\Delta \cap W_{1}=\varnothing$. From now on, we fix such a sequence $\left\{W_{n}\right\}$.

We describe now how we will continuously embed $E_{0}$ into $E$. We fix winning strategies $\Sigma_{1}=\left\{f_{n}^{1}\right\}$ and $\Sigma_{2}=\left\{f_{n}^{2}\right\}$ for player $\alpha$ in the strong Choquet games corresponding to $(\mathscr{N}, \tau)$ and $\left(\mathscr{N}^{2}, \tau_{2}\right)$ respectively.

By the remark at the end of $\S 2$, we may assume that for the usual distance on $\mathcal{N}, \operatorname{diam}\left(f_{n}^{1}\left(U_{0}, x_{0}, \ldots, U_{n-1}, x_{n-1}\right)\right) \leq 1 /(n+1)$, and similarly for $\Sigma_{2}=$ $\left\{f_{n}^{2}\right\}$.

We will define, for each binary sequence $s \in 2^{<\omega} \quad(s \neq \varnothing)$ a point $x_{s} \in X$, and two $\tau$-open sets $U_{s}, \mathbf{U}_{s}$ containing $x_{s}, U_{s}, \mathbf{U}_{s} \subseteq X$, such that

(i) If $s$ is of length $n, \mathbf{U}_{s}=f_{n}^{1}\left(U_{s(0)}, x_{s(0)}, \ldots, U_{s}, x_{s}\right)$, i.e., the play

$$
\begin{array}{cc}
\underline{\beta} & \underline{\alpha} \\
U_{s(0)}, x_{s(0)} & \mathbf{U}_{s(0)} \\
U_{s(0), s(1)}, x_{s(0), s(1)} & \\
\vdots & \mathbf{U}_{s(0), s(1)} \\
U_{s}, x_{s} & \\
& \mathbf{U}_{s}
\end{array}
$$

is a play in the strong Choquet game for $\tau$, where $\alpha$ answers by his winning strategy $\Sigma_{1}$;

(ii) for all $s, t$ in $2^{n}, x_{s} \bar{E} x_{t}$; and

(iii) if $s=\widehat{s_{1}} 0$ and $t=\widehat{t_{1}} \hat{1}$, then

$$
U_{s} \times U_{t} \subseteq W_{n}
$$

We will also define, for all $k \in \omega$ and $s \in 2^{m}$, nonempty $\tau_{2}$-open sets $E_{k, s} \mathbf{E}_{k, s}$ such that

(iv) $E_{k, \varnothing}=E$;

(v) $\left(x_{0^{k} \hat{0} \hat{s}_{s}}, x_{0^{k} \hat{1}_{1} \hat{s}}\right) \in E_{k, s} ;$ and

(vi) $\mathbf{E}_{k, s}=f_{m}^{2}\left(E_{k, \varnothing},\left(x_{0^{k}{ }_{0}}, x_{0^{k} \wedge_{1}}\right), E_{k, s(0)},\left(x_{0^{k} \hat{0}^{\wedge} s(0)}, x_{0^{k} \wedge_{1} s_{(0)}}\right), \ldots\right.$,

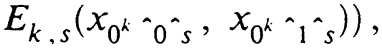


i.e., the play

$$
\begin{aligned}
& \underline{\beta} \quad \underline{\alpha} \\
& E_{k, \varnothing},\left(x_{0^{k} \wedge_{0}}, x_{0^{k} \wedge_{1}}\right) \\
& \begin{array}{ll}
E_{k, s(0)},\left(x_{0^{k} \wedge \hat{0} s(0)}, x_{0^{k} \wedge \hat{\wedge} s(0)}\right) & \mathbf{E}_{k, \varnothing} \\
\mathbf{E}_{k, s(0)}
\end{array} \\
& E_{k, s},\left(x_{0^{k} \hat{0} \wedge s}, x_{0^{k} \hat{1} \wedge}\right) \\
& \mathbf{E}_{k, s}
\end{aligned}
$$

is a play in the strong Choquet game for $\tau_{2}$, in which player $\alpha$ follows his winning strategy $\Sigma_{2}$.

Suppose this construction has been carried out. For each $\gamma \in 2^{\omega}$, the sequence $\left(U_{\gamma \mid n}, x_{\gamma \mid n}, \mathbf{U}_{\gamma \mid n}\right)$ is by (i) a winning play for player $\alpha$ in the strong Choquet game for $\tau$, hence $\bigcap_{n} U_{\gamma / n}$ consists of a single point which we denote by $f(\gamma)$. By the property on the diameters of the $\mathbf{U}_{s}$ 's, $f$ is continuous. Also $f$ is one-to-one, for if $\gamma \neq \gamma^{\prime}$ and $n$ is the first place where $\gamma(n) \neq \gamma^{\prime}(n)$, say $\gamma(n)=0$ and $\gamma^{\prime}(n)=1$, then by (iii) $U_{\gamma \mid n+1} \times U_{\gamma^{\prime} \mid n+1} \subseteq W_{n+1} \subseteq W_{1}$, so in particular, as $\Delta \cap W_{1}=\varnothing, U_{\gamma \nmid n+1} \cap U_{\gamma^{\prime} \mid n+1}=\varnothing$.

We claim that $f$ works, i.e., that

$$
\gamma E_{0} \delta \leftrightarrow f(\gamma) E f(\delta)
$$

Suppose first that $\gamma \mathbb{E}_{0} \delta$. Then say for infinitely many $n, \gamma(n)=0$ and $\delta(n)=$ 1. Again by (iii) one gets for such $n$

$$
U_{y \mid n+1} \times U_{\delta\lceil n+1} \subseteq W_{n+1}
$$

and hence $(f(\gamma), f(\delta)) \in W_{n+1}$. As the sequence $\left\{W_{n}\right\}$ is decreasing, this means that

$$
(f(\gamma), f(\delta)) \in \bigcap_{n} W_{n}
$$

so that $f(\gamma) f(\delta)$. Conversely, we show that for all $s, t \in 2^{k}$ and $\gamma \in 2^{\omega}$, $(f(\widehat{s} \gamma), f(\hat{t} y)) \in E$. This is proved by induction on $k$. This is trivial if $k=0$, i.e., $s=t=\varnothing$. So assume it has been proved for $k$, and consider $s=\widehat{s_{1}} i, t=\hat{t_{1}} j$ with $s_{1}, t_{1} \in 2^{k}$ and $i, j$ in $\{0,1\}$. If $i=j$, then $\widehat{s} \gamma=$ $\widehat{s_{1}}\left(\hat{i^{\gamma} \gamma}\right)$ and $\hat{t} \gamma=\hat{t_{1}}(\hat{i} \gamma)$ so the induction hypothesis applies to give the result. So it remains to consider the case say $i=0$ and $j=1$. By the induction

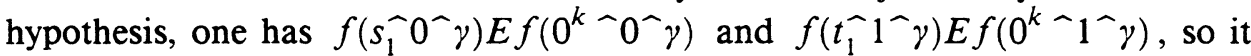
is enough to show that $f\left(0^{k}{ }^{-} 0^{\wedge} \gamma\right) E\left(0^{k}{ }^{\wedge}{ }^{\wedge} \gamma\right)$. By property (vi), the sequence $\left(E_{k, \gamma \mid n},\left(x_{0^{k} \hat{0}_{\gamma \mid n}}, x_{0^{k} \hat{1}_{\gamma \mid n}}\right), \mathbf{E}_{k, \gamma \mid n}\right)$ is a winning play for player $\alpha$ in the strong Choquet game for $\tau_{2}$, hence $\bigcap_{n} E_{k, \gamma \mid n}$ consists of a single point, which must be the point $\left(f\left(0^{k}{ }^{-} 0^{-} \gamma\right), f\left(0^{k}{ }^{-}{ }^{-} \gamma\right)\right)$. And as by (iv) $E_{k, \varnothing}=E$ we get $f\left(0^{k}{ }^{\wedge} \gamma\right) E f\left(0^{k} \wedge 1^{\wedge} \gamma\right)$ as desired. 
We go now to the main construction.

First we have to find $x_{0}, x_{1}$ in $X, U_{0}, U_{1} \tau$-open neighborhoods of $x_{0}, x_{1}$ with $U_{0} \times U_{1} \subseteq W_{1}$, and (as $\left.E_{0, \varnothing}=E\right)\left(x_{0}, x_{1}\right) \in E$. This is possible, as $W_{1} \cap \bar{E}$ is dense open in $\left(X^{2} \cap \bar{E}, \tau \times \tau\right)$, and $E$ is dense in this set. We then let $\mathbf{U}_{0}=f_{1}^{1}\left(U_{0}, x_{0}\right), \mathbf{U}_{1}=f_{1}^{1}\left(U_{1}, x_{1}\right)$, and $\mathbf{E}_{0, \varnothing}=f_{1}^{2}\left(E_{0, \varnothing},\left(x_{0}, x_{1}\right)\right)$.

Suppose the construction has been done, satisfying (i)-(vi), for all sequences in $2^{n}$, and all $k, u \in 2^{m}$ with $k+1+m=n$ (i.e., $0^{k}{ }^{\wedge} s$ and $0^{k} \wedge \uparrow s$ also of length $n)$. We now do it for $n+1$. Consider the space $\bar{E}^{2^{n}} \times \bar{E}^{2^{n}}$ with the $\tau_{2^{n}} \times \tau_{2^{n}}$ topology, the elements of which are tuples $\bar{y}=\left(\left(y_{s}{ }_{0}\right)_{s \in 2^{n}},\left(y_{s_{1}{ }_{1}}\right)_{s \in 2^{n}}\right)$, and the open subset of it:

$$
\begin{aligned}
& Y_{n}=\left\{\bar{y}: \forall s \in 2^{n}\left(y_{s \hat{\nu}_{0}} \in \mathbf{U}_{s} \text { and } y_{s_{1}} \in \mathbf{U}_{s}\right)\right. \text { and } \\
& \forall k \forall u \in 2^{m} \text { with } k+1+m=n \\
& \left(y_{0^{k} \hat{0} \hat{u} \hat{0}}, y_{0^{k} \hat{1} \hat{u} \hat{0}}\right) \in \mathbf{E}_{k, u} \text { and } \\
& \left.\left(y_{0^{k} \hat{0^{\prime}} \hat{u} 1}, y_{0^{k} \hat{1} \hat{u_{1}}}\right) \in \mathbf{E}_{k, u}\right\} \text {. }
\end{aligned}
$$

That $Y_{n}$ is $\left(\tau_{2^{n}} \times \tau_{2^{n}}\right)$-open follows from the fact that $Y_{n}$ is a square-the conditions on the $\left(y_{s{ }_{0}}\right)_{s \in 2^{n}}$ and the $\left(y_{s{ }_{1}}\right)_{s \in 2^{n}}$ being the same $\tau_{2^{n}}$-open conditions. Note that the sequence $\left(\left(x_{s}\right)_{s \in 2^{n}},\left(x_{s}\right)_{s \in 2^{n}}\right)$ is in $Y_{n}$, by the induction hypothesis, so $Y_{n} \neq \varnothing$.

Now for all $s, t \in 2^{n}$, the projection map $\bar{y} \mapsto\left(y_{s_{0} 0}, y_{\hat{t}_{1}}\right)$ is open from $\left(\bar{E}^{2^{n}} \times \bar{E}^{2^{n}}, \tau_{2^{n}} \times \tau_{2^{n}}\right)$ into $(\bar{E}, \tau \times \tau)$, and as $W_{n+1} \cap \bar{E}$ is $(\tau \times \tau)$-dense open in $X^{2} \cap \bar{E}$, the set

$$
V_{n}=\left\{\bar{y} \in Y_{n}: \forall s \in 2^{n} \forall t \in 2^{n}\left(y_{s^{\hat{0}}}, y_{\hat{i}_{1}}\right) \in W_{n+1}\right\}
$$

is open and nonempty in $\tau_{2^{n}} \times \tau_{2^{n}}$. But then the set

$$
V_{n}^{*}=\left\{\left(y_{0^{n} \hat{\imath}_{0}}, y_{0^{n} \hat{\imath}_{1}}\right): \exists\left(y_{s_{0} \hat{0}}, y_{s_{1} \hat{1}_{1}}\right)_{s \in 2^{n}, s \neq 0^{n}}\left(\bar{y} \in V_{n}\right)\right\}
$$

is a nonempty $(\tau \times \tau)$-open set in $\bar{E} \cap X^{2}$, hence must meet the dense set $E$. This means that we can find a family $\left(x_{s \hat{0}}\right)_{s \in 2^{n}},\left(x_{s \hat{1}}\right)_{s \in 2^{n}}$ such that:

(a) $x_{s{ }_{i}{ }_{i}} \overline{x_{t} j}$, for all $s, t \in 2^{n}, i, j \in 2$,

(b) $x_{s{ }_{0}} \in \mathbf{U}_{s}, x_{s{ }_{1}} \in \mathbf{U}_{s}$, for all $s \in 2^{n}$,

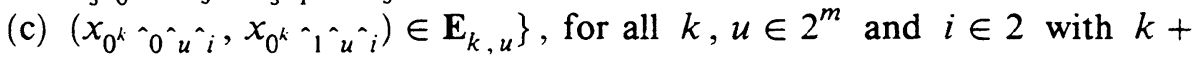
$1+m=n$

(d) $\left(x_{s_{0} \hat{0}}, x_{\hat{t}_{1}}\right) \in W_{n+1}$, for all $s, t \in 2^{n}$,

(e) $\left(x_{0^{n} \hat{n}_{0}}, x_{0^{n} \wedge_{1}}\right) \in E$.

This family is the one we were searching for. It satisfies (ii) by property (a). By properties (b) and (d), as $W_{n+1}$ is $(\tau \times \tau)$-open, we can find neighborhoods

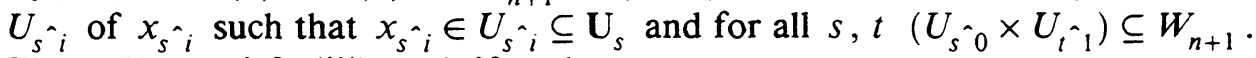
These $U_{s i} i$ satisfy (iii), and, if we let

$$
\mathbf{U}_{s_{i} i}=f_{n+1}^{1}\left(U_{s(0)}, x_{s \hat{0}}, \ldots, U_{s \hat{i}}, x_{s_{i} i}\right) \text {, }
$$


(i) is also satisfied. By (c), we can define $E_{k, u_{i} i}$, for $u \in 2^{m}$ with $k+1+$ $m=n$, by $E_{k, u_{i}}=\mathbf{E}_{k, u}$, so that (v) is satisfied, and then let $\mathbf{E}_{k, u_{i}}=$

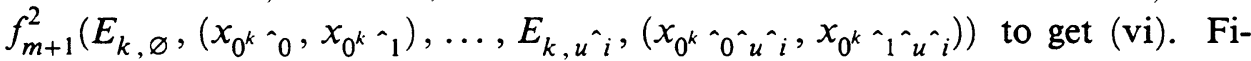
nally by (e) we let $E_{n, \varnothing}=E$ to get (iv), and it satisfies (v), and if we let $\mathbf{E}_{n, \varnothing}=f_{1}^{2}\left(E_{n, \varnothing},\left(x_{0^{n} \hat{\wedge}_{0}}, x_{0^{n} \wedge_{1}}\right)\right)$, we also get (vi) for it, so that the proof is finished.

Let us make some final comments on the combinatorial aspects of this proof: Properties (b) and (c) above are open, and independent in the blocks $\left(x_{s}{ }_{0}\right)_{s \in 2^{n}}$ and $\left(x_{s}{ }_{1}\right)_{s \in 2^{n}}$, the linking conditions (d) are open dense, while the crucial linking condition (e) is only dense. This works with one such condition, but it would not work with two of them. This is why in the proof we only put the weak conditions (iv), (v), and (vi), and had to give an argument to show that they are enough, using the transitivity of $E$, to get $f\left(s^{\wedge} \gamma\right) E f\left(t^{\wedge} \gamma\right)$ for all $s, t$ of the same length, and not only for $f\left(0^{k}{ }^{-} 0^{-} \gamma\right)$ and $f\left(0^{k}{ }^{-}{ }^{-} \gamma\right)$. Note that the same argument shows that a posteriori one necessarily has $x_{s} E x_{t}$ for all $s, t$ of the same length, but this could not have been asked for a priori.

\section{REFERENCES}

[B1] J. P. Burgess, Equivalences generated by families of Borel sets, Proc. Amer. Math. Soc. 69 (1978), 323-326.

[B2] _ A selection theorem for group actions, Pacific J. Math. 80 (1979), 333-336.

[D] J. Dixmier, C*-algebras, North-Holland, 1977.

[DJK1] R. Dougherty, S. Jackson, and A. Kechris, The structure of equivalence relations on Polish spaces, I: An extension of the Glimm-Effros dichotomy, circulated notes, 1989.

[DJK2] _ _, The structure of equivalence relations on Polish spaces, II: Countable equivalence relations or descriptive dynamics, circulated notes, 1989.

[E1] E. G. Effros, Transformation groups and $C^{*}$-algebras, Ann. of Math. 81 (1965), 38-55.

[E2] _ Polish transformation groups and classification problems, General Topology and Modern Analysis (Rao and Mc Auley, eds.), Academic Press, 1980, pp. 217-227.

[Gl1] J. Glimm, Type I $C^{*}$-algebras, Ann. of Math. 73 (1961), 572-612.

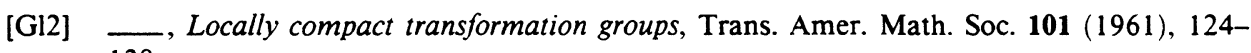
138.

[Go] G. Godefroy, Some remarks on Suslin sections, Fund. Math. 84 (1986), 159-167.

[HMS] L. Harrington, D. Marker, and S. Shelah, Borel orderings, Trans. Amer. Math. Soc. 310 (1988), 293-302.

[Ka] K. Kada, $A$ Borel version of Dilworth's theorem (to appear).

[KW] Y. Katznelson and B. Weiss, The construction of quasi-invariant measures, Israel J. Math. 12 (1972), 1-4.

[Ke] A. S. Kechris, Amenable equivalence relations and Turing degrees, J. Symbolic Logic (to appear).

[Krl] W. Krieger, On quasi-invariant measures on uniquely ergodic systems, Invent. Math. 14 (1971), 184-196.

[Kr2] _ On Borel automorphisms and their quasi-invariant measures, Math. Z. 151 (1976), 19-24.

[Ku] K. Kuratowski, Topology, vol. I, Academic Press, 1966.

[L] A. Louveau, Two results on Borel orders, J. Symbolic Logic 34 (1989), 865-874. 
[LS] A. Louveau and J. Saint Raymond, On the quasi-ordering of Borel linear orders under embeddability, J. Symbolic Logic 55 (1990), 537-560.

[MK] D. A. Martin and A. S. Kechris, Infinite games and effective descriptive set theory, Analytic Sets (C. A. Rogers et al., eds.), Acadenic Press, 1980.

[M] Y. Moschovakis, Descriptive set theory, North-Holland, 1980.

[SW] S. Shelah and B. Weiss, Measurable recurrence and quasi-invariant measures, Israel J. Math. 43 (1982), 154-160.

[S] J. H. Silver, Counting the number of equivalence classes of Borel and coanalytic equivalence relations, Ann. Math. Logic 18 (1980), 1-28.

[U] C. Uzcategui, Ph.D. Thesis, California Institute of Technology, 1990.

[W] B. Weiss, Measurable dynamics, Contemporary Math. 26 (1984), 395-421.

[Z] R. J. Zimmer, Ergodic theory and semisimple groups, Birkhäuser, 1984.

Department of Mathematics, University of California, Berkeley, California 94720

Department of Mathematics, California Institute of Technology, Pasadena, CaliFORNIA 91125

Equipe d’Analyse, Université Paris Vi, 4, Place Jussieu, 75230 Paris Cedex 05, France 